(n)

trobertivier Journal of Nonlinear Mathematical Physics

\title{
A Perturbative Approach for the Asymptotic Evaluation of the Neumann Value Corresponding to the Dirichlet Datum of a Single Periodic Exponential for the NLS
}

Guenbo Hwang

To cite this article: Guenbo Hwang (2014) A Perturbative Approach for the Asymptotic Evaluation of the Neumann Value Corresponding to the Dirichlet Datum of a Single Periodic Exponential for the NLS, Journal of Nonlinear Mathematical Physics 21:2, 225247, DOI: https://doi.org/10.1080/14029251.2014.905298

To link to this article: https://doi.org/10.1080/14029251.2014.905298

Published online: 04 January 2021 


\title{
A Perturbative Approach for the Asymptotic Evaluation of the Neumann Value Corresponding to the Dirichlet Datum of a Single Periodic Exponential for the NLS
}

\author{
Guenbo Hwang \\ Department of Mathematics, Daegu University, \\ Gyeongsan Gyeongbuk, 712-714, Korea \\ ghwang@daegu.ac.kr
}

Received 3 December 2013

Accepted 3 February 2014

\begin{abstract}
Boundary value problems for the nonlinear Schrödinger equation formulated on the half-line can be analyzed by the Fokas method. For the Dirichlet problem, the most difficult step of this method is the characterization of the unknown Neumann boundary value. For the case that the Dirichlet datum consists of a single periodic exponential, namely, $a \exp (i \omega t), a, \omega$ real, it has been shown in [2-4] that if one assumes that the Neumann boundary value is given for large $t$ by $c \exp (i \omega t)$, then $c$ can be computed explicitly in terms of $a$ and $\omega$. Here, using the perturbative approach introduced in [16], it is shown that for typical initial conditions, it is indeed the case that at least up to third order in a perturbative expansion the Neumann boundary value is given by $c \exp (i \omega t)$ and the value of $c$ is at least up to this order the value found in [2-4].
\end{abstract}

Keywords: Initial-boundary value problem; Generalized Dirichlet to Neumann map; Nonlinear Schrödinger equation.

2000 Mathematics Subject Classification: 47K15, 35Q55

\section{Introduction}

A unified method for analyzing boundary value problems, extending ideas of the so-called inverse scattering transform method, was introduced in [5], see also [6] and the review [7] (as well as the review [12] for the implementation of the unified method to linear PDEs).

For integrable nonlinear evolution PDEs, the most difficult step in the implementation of this so-called Fokas method, is the characterization of the Dirichlet to Neumann map. For example, for the nonlinear Schrödinger equation (NLS) on the half-line with given initial $q(x, 0)=q_{0}(x)$ and Dirichlet $q(0, t)=g_{0}(t)$ data, this involves characterizing the unknown Neumann boundary value $q(0, t)=g_{1}(t)$ in terms of $q_{0}(x)$ and $g_{0}(t)$. In this respect we note: (i) for certain particular boundary conditions called linearizable, the above characterization can be achieved via explicit formulas and hence for these cases the Fokas method is as effective as the inverse scattering transform method. (ii) If $g_{0}(t)$ vanishes as $t \rightarrow \infty$, it is possible to bypass the characterization of the Dirichlet to Neumann map and to obtain the asymptotic form of the solution as $t \rightarrow \infty[8-10,15]$. (iii) If $g_{0}(t)$ is a periodic function, then in order to obtain the large $t$ asymptotics of the solution $q(x, t)$, it is first necessary to determine the asymptotic form of $g_{1}(t)$ as $t \rightarrow \infty$.

Pioneering results regarding (iii) have been obtained in a series of papers by Boutet de Monvel and co-authors [2-4]. The final result of these authors is the following: Consider the NLS on the 
half-line

$$
i q_{t}+q_{x x}-2 \lambda|q|^{2} q=0, \quad x>0, \quad t>0,
$$

with $\lambda= \pm 1$ and a single periodic exponential as the Dirichlet datum,

$$
q(0, t)=a e^{i \omega t}, \quad a, \omega \text { real. }
$$

Assume that the Neumann boundary value is asymptotically periodic, namely,

$$
q_{x}(0, t)=c e^{i \omega t}+o(1), \quad t \rightarrow \infty .
$$

Then, $c$ can be obtained explicitly in terms of $a$ and $\omega$ :

$$
\begin{array}{ll}
\omega>0: & c=-a \sqrt{\omega+\lambda a^{2}}, \\
\omega<0: & c=i a \sqrt{-\omega-2 \lambda a^{2}} .
\end{array}
$$

An effective characterization of the Dirichlet to Neumann map for the NLS equation on the half-line was recently presented in [11] and [16] (see also $[13,14]$ for the application of the sineGordon and the modified Korteweg-de Vries equations formulated on the half-line) using two different formulations, both of which are based on the analysis of the global relation: the formulation in [11] is based on the eigenfunctions involved in the definition of the spectral functions $\{A(k), B(k)\}$, whereas the formulation in [16] is based on an extension of the Gelfand-LevitanMarchenko approach first introduced in [1]. In particular, in [16] a perturbative approach was introduced for the explicit construction of the Neumann boundary value as $t \rightarrow \infty$. Using this approach, we show here that for the Dirichlet datum (1.2) and the initial datum

$$
q(x, 0)=a e^{-\eta x}, \quad \eta>0, \quad 0<x<\infty,
$$

the Neumann boundary value is indeed given up to third order in $a$ by (1.3), where $c$ satisfies, at least up to this order, equations (1.4).

\section{The Main Result}

Theorem 2.1. Let

$$
q(x, t)=\varepsilon q_{1}(x, t)+\varepsilon^{2} q_{2}(x, t)+\cdots, \quad \varepsilon \rightarrow 0,
$$

be the perturbation solution $q(x, t)$ of the NLS on the half-line with the initial data

$$
q(x, 0)=\varepsilon e^{-\eta x}, \quad \eta>0
$$

and the Dirichlet boundary data

$$
q(0, t)=\varepsilon g_{01}(t)+O\left(\varepsilon^{4}\right), \quad \varepsilon \rightarrow 0,
$$

where

$$
g_{01}(t)=e^{i \omega t}, \quad \omega \in \mathbb{R} .
$$

Then,

$$
q_{x}(0, t)=\varepsilon g_{11}(t)+\varepsilon^{3} g_{13}(t)+O\left(\varepsilon^{4}\right), \quad \varepsilon \rightarrow 0,
$$

where $g_{11}$ and $g_{13}$ are given by the following formulas: 
(i) $\omega>0$

$$
\begin{aligned}
& g_{11}(t)=-\sqrt{\omega} e^{i \omega t}+o(1), \quad t \rightarrow \infty, \\
& g_{13}(t)=-\frac{\lambda}{2 \sqrt{\omega}} e^{i \omega t}+o(1), \quad t \rightarrow \infty .
\end{aligned}
$$

(ii) $\omega<0$

$$
\begin{aligned}
& g_{11}(t)=i \sqrt{-\omega} e^{i \omega t}+o(1), \quad t \rightarrow \infty, \\
& g_{13}(t)=-\frac{i \lambda}{\sqrt{-\omega}} e^{i \omega t}+o(1), \quad t \rightarrow \infty .
\end{aligned}
$$

Proof. It is shown in [11] that

$$
\begin{aligned}
& q_{x}(0, t)=\frac{2}{i \pi} \int_{\partial D_{3}}\left[i q(0, t)+k\left(\Phi_{1}(t, k)-\Phi_{1}(t,-k)\right)\right] d k \\
& \quad+\frac{2 q(0, t)}{\pi} \int_{\partial D_{3}}\left(\Phi_{2}(t, k)-\Phi_{2}(t,-k)\right) d k-\frac{4}{i \pi} \int_{\partial D_{3}} k e^{-4 i k^{2} t} \frac{b(-k)}{a(-k)} \overline{\Phi_{2}(t,-\bar{k})} d k,
\end{aligned}
$$

where $\partial D_{3}$ is the oriented boundary of the third quadrant of the complex $k$-plane and $\left\{\Phi_{1}, \Phi_{2}\right\}$ solve the following system of equations:

$$
\begin{aligned}
& \Phi_{1}(t, k)=\int_{0}^{t} e^{4 i k^{2}\left(t-t^{\prime}\right)}\left[-i \lambda\left|q\left(0, t^{\prime}\right)\right|^{2} \Phi_{1}\left(t^{\prime}, k\right)+\left(2 k q\left(0, t^{\prime}\right)+i q_{x}\left(0, t^{\prime}\right)\right) \Phi_{2}\left(t^{\prime}, k\right)\right] d t^{\prime}, \\
& \Phi_{2}(t, k)=1+\lambda \int_{0}^{t}\left[\left(2 k \bar{q}\left(0, t^{\prime}\right)-i \bar{q}_{x}\left(0, t^{\prime}\right)\right) \Phi_{1}\left(t^{\prime}, k\right)+i\left|q\left(0, t^{\prime}\right)\right|^{2} \Phi_{2}\left(t^{\prime}, k\right)\right] d t^{\prime} .
\end{aligned}
$$

Substituting in the above equation (2.2) and (2.4), as well as (2.7b) and (2.7c), we find the following equations:

$$
\begin{aligned}
g_{11}(t)= & \frac{2}{i \pi} \int_{\partial D_{3}}\left\{k\left[\Phi_{11}(t, k)-\Phi_{11}(t,-k)\right]+i g_{01}(t)\right\} d k-\frac{4}{i \pi} \int_{\partial D_{3}} k e^{-4 i k^{2} t} b_{1}(-k) d k \\
g_{13}(t)= & \frac{2}{i \pi} \int_{\partial D_{3}} k\left[\Phi_{13}(t, k)-\Phi_{13}(t,-k)\right] d k+\frac{2}{\pi} g_{01}(t) \int_{\partial D_{3}}\left[\Phi_{22}(t, k)-\Phi_{22}(t,-k)\right] d k \\
& -\frac{4}{i \pi} \int_{\partial D_{3}} k e^{-4 i k^{2} t} b_{1}(-k)\left[\overline{\Phi_{22}(t,-\bar{k})}-a_{2}(-k)\right] d k \\
& \Phi_{11}(t, k)=e^{-4 i k^{2} t} \int_{0}^{t} e^{4 i k^{2} \tau}\left(2 k g_{01}(\tau)+i g_{11}(\tau)\right) d \tau, \\
\lambda \Phi_{22}(t, k)= & \int_{0}^{t}\left[2 k \bar{g}_{01}(\tau)-i \bar{g}_{11}(\tau)\right] \Phi_{11}(\tau, k) d \tau+i \int_{0}^{t}\left|g_{01}(\tau)\right|^{2} d \tau, \\
\lambda & \Phi_{13}(t, k)=e^{-4 i k^{2} t} \int_{0}^{t} e^{4 i k^{2} \tau}\left[-i\left|g_{01}\right|^{2} \Phi_{11}(\tau, k)+\left(2 k g_{01}+i g_{11}\right) \lambda \Phi_{22}(\tau, k)\right. \\
& \left.+i \lambda g_{13}(\tau)\right] d \tau,
\end{aligned}
$$

where the functions $b_{1}(k)$ and $a_{2}(k)$ are given by

$$
\begin{aligned}
& b_{1}(k)=-\int_{0}^{\infty} e^{2 i k x} e^{-\eta x} d x, \\
& a_{2}(k)=\lambda \int_{0}^{\infty} e^{-\eta x} \int_{x}^{\infty} e^{-2 i k\left(x-x^{\prime}\right)} e^{-\eta x^{\prime}} d x^{\prime} d x .
\end{aligned}
$$



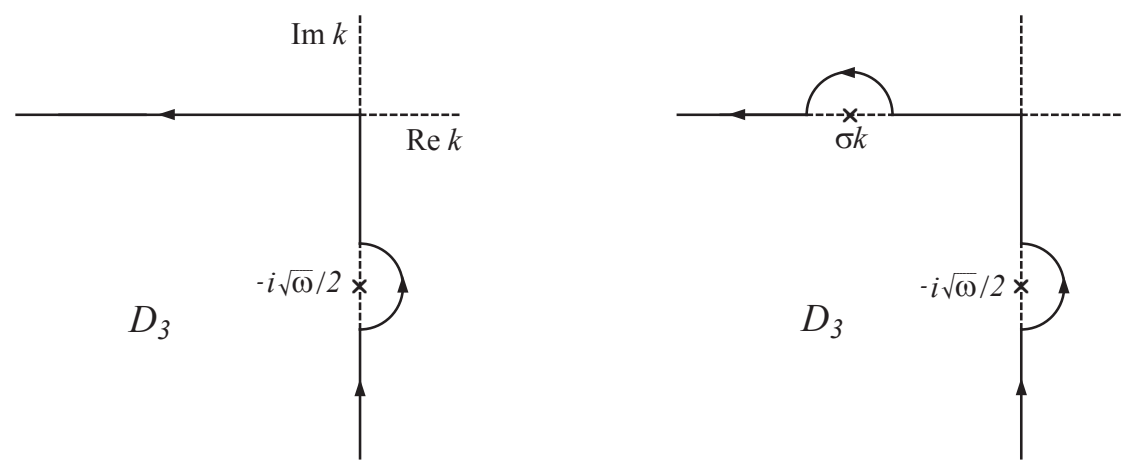

Fig. 1. $(\omega>0)$ The deformed contours $\partial \tilde{D}_{3}$ (left) and $\partial \tilde{D}_{3}^{\sigma k}$ (right).

The proof of of Theorem 2.1 is the consequence of several propositions and lemmas given below in Sections 3 and 4.

\section{A Perturbative Approach for $\omega>0$}

Proposition 3.1. For $\omega>0$,

$$
g_{11}(t)=-\sqrt{\omega} e^{i \omega t}+\int_{\partial \tilde{D}_{3}} e^{-4 i k_{3}^{2} t} A\left(k_{3}^{2}\right) d k_{3}+\frac{4}{i \pi} \int_{\partial D_{3}} \frac{k e^{-4 i k^{2} t}}{2 i k+\eta} d k,
$$

where the contour $\partial \tilde{D}_{3}$ is depicted in figure 1 and

$$
A\left(k^{2}\right)=\frac{8 k^{2}}{\pi\left(4 k^{2}+\omega\right)} .
$$

Furthermore,

$$
\int_{\partial \tilde{D}_{3}} e^{-4 i k_{3}^{2} t} A\left(k_{3}^{2}\right) d k_{3}=O\left(t^{-3 / 2}\right), \quad t \rightarrow \infty,
$$

and

$$
\int_{\partial D_{3}} \frac{k e^{-4 i k^{2} t}}{2 i k+\eta} d k=O\left(t^{-3 / 2}\right), \quad t \rightarrow \infty
$$

Proof. Using (2.13), we find

$$
b_{1}(-k)=-\int_{0}^{\infty} e^{-(2 i k+\eta) x} d x=-\frac{1}{2 i k+\eta},
$$

and then substituting (3.4) into (2.8), we obtain the last terms in (3.1).

Note that equation (2.10) yields

$$
k\left[\Phi_{11}(t, k)-\Phi_{11}(t,-k)\right]=-\frac{4 i k^{2}}{4 k^{2}+\omega}\left(e^{i \omega t}-e^{-4 i k^{2} t}\right) .
$$

Inserting the rhs of (3.5) into the rhs of (2.8), we find

$$
\frac{2}{i \pi} \int_{\partial D_{3}}\left\{k\left[\Phi_{11}(t, k)-\Phi_{11}(t,-k)\right]+i g_{01}(t)\right\} d k=\frac{2}{\pi} \int_{\partial D_{3}} \frac{4 k^{2} e^{-4 i k^{2} t}+\omega e^{i \omega t}}{4 k^{2}+\omega} d k .
$$


Note that the integrand in the rhs of (3.6) has removable singularities at $k=-i \sqrt{\omega} / 2$. In what follows it will be necessary to split the integral in (3.6) in two separate integrals. Thus, before splitting we deform the contour $\partial D_{3}$ to the contour $\partial \tilde{D}_{3}$. Hence, (3.6) becomes

$$
g_{11}(t)=\frac{2}{\pi} \int_{\partial \tilde{D}_{3}} \frac{\omega e^{i \omega t}}{4 k^{2}+\omega} d k+\frac{2}{\pi} \int_{\partial \tilde{D}_{3}} \frac{4 k^{2} e^{-4 i k^{2} t}}{4 k^{2}+\omega} d k
$$

The above splitting is consistent with the fact that $e^{-4 i k^{2} t}$ decays in the second and fourth quadrants of the complex $k$-plane as $t \rightarrow \infty$. Using the residue theorem to compute the first integral in the rhs of (3.7), we find the first two terms in (3.1).

It is straightforward to estimate the large $t$ behavior of the integrals appearing in the rhs of (3.1). Indeed, let us denote the integral in (3.1) by $\frac{8}{\pi} I$. Using the Cauchy theorem, we deform the part of $\partial \tilde{D}_{3}$ involving the negative real axis along the ray $\arg k=3 \pi / 4$, which we denote by $\tilde{C}_{1}$ (cf. figure3), and along $C_{\infty}$, where $C_{\infty}$ is the limit of $C_{R}=\{|k|=R, 3 \pi / 4 \leq \arg k \leq \pi\}$ as $R \rightarrow \infty$ :

$$
I=\left(\int_{\tilde{C}_{1}}+\int_{C_{\infty}}\right) \frac{k^{2} e^{-4 i k^{2} t}}{4 k^{2}+\omega} d k
$$

For the first integral in (3.8), letting $k=\rho e^{3 i \pi / 4}$, we find

$$
i e^{3 i \pi / 4} \int_{0}^{\infty} \frac{\rho^{2} e^{-4 \rho^{2} t} d \rho}{4 i \rho^{2}-\omega}=-i e^{-i \pi / 4} \int_{0}^{\infty} \frac{\rho^{2} e^{-4 \rho^{2} t} d \rho}{4 i \rho^{2}-\omega} .
$$

For the second integral in (3.8), let $k=R e^{i \theta}$ with $3 \pi / 4 \leq \theta \leq \pi$. Thus, for large $R$, we obtain

$$
\left|\int_{C_{R}} \frac{k^{2} e^{-4 i k^{2} t}}{4 k^{2}+\omega} d k\right| \leq \int_{3 \pi / 4}^{\pi} \frac{R^{3} e^{4 R^{2} t \sin 2 \theta}}{4 R^{2}-|\omega|} d \theta=\frac{\pi R^{3}}{16 R^{2}-4|\omega|}\left[I_{0}\left(4 R^{2} t\right)-L_{0}\left(4 R^{2} t\right)\right],
$$

where $I_{n}(z)$ is the modified Bessel function and $L_{n}(z)$ is the modified Struve function. Using the asymptotics

$$
I_{0}(z)-L_{0}(z) \sim-\frac{2}{\pi z}, \quad|z| \rightarrow \infty
$$

we find that the rhs of (3.10) vanishes as $R \rightarrow \infty$ and hence the second integral in (3.8) is identically zero.

Similarly, we can show that the integral along the part of $\partial \tilde{D}_{3}$ involving the negative imaginary axis yields the same expression as in (3.9). Thus,

$$
\int_{\partial \tilde{D}_{3}} \frac{k^{2} e^{-4 i k^{2} t}}{4 k^{2}+\omega} d k=-2 i e^{-i \pi / 4} \int_{0}^{\infty} \frac{\rho^{2} e^{-4 \rho^{2} t} d \rho}{4 i \rho^{2}-\omega} .
$$

A stationary point calculation implies that the leading order contribution vanishes. The leading order contribution from integration by parts also vanishes, thus we obtain (3.3a). Equation (3.3b) can be derived in a similar way. 
G. Hwang

We write $g_{11}(t)$ as

$$
g_{11}(t)=g_{11}^{(1)}(t)+g_{11}^{(2)}(t)
$$

where

$$
g_{11}^{(1)}(t)=-\sqrt{\omega} e^{i \omega t}+\int_{\partial \tilde{D}_{3}} e^{-4 i k_{3}^{2} t} A\left(k_{3}^{2}\right) d k_{3}
$$

and

$$
g_{11}^{(2)}(t)=\frac{4}{i \pi} \int_{\partial D_{3}} \frac{k e^{-4 i k^{2} t}}{2 i k+\eta} d k
$$

Also, we denote $\Phi_{11}(t, k)$ by

$$
\Phi_{11}(t, k)=\Phi_{11}^{(1)}(t, k)+\Phi_{11}^{(2)}(t, k)
$$

where

$$
\Phi_{11}^{(1)}(t, k)=e^{-4 i k^{2} t} \int_{0}^{t} e^{4 i k^{2} \tau}\left(2 k g_{01}(\tau)+i g_{11}^{(1)}(\tau)\right) d \tau
$$

and

$$
\Phi_{11}^{(2)}(t, k)=i e^{-4 i k^{2} t} \int_{0}^{t} e^{4 i k^{2} \tau} g_{11}^{(2)}(\tau) d \tau
$$

Lemma 3.1. For $\omega>0$,

$$
\Phi_{11}^{(1)}(t, k)=\frac{i\left(e^{-4 i k^{2} t}-e^{i \omega t}\right)}{2 k+i \sqrt{\omega}}+\frac{i e^{-4 i k^{2} t}}{2 \sigma k-i \sqrt{\omega}}+\frac{1}{4} \int_{\partial \tilde{D}_{3}^{\sigma \sigma}} \frac{e^{-4 i k_{3}^{2} t}}{k^{2}-k_{3}^{2}} A\left(k_{3}^{2}\right) d k_{3},
$$

where the contour $\partial \tilde{D}_{3}^{\sigma k}$ is depicted in figure 1 and $\sigma=1$ if $k \in \partial D_{3}$, whereas $\sigma=-1$ if $-k \in \partial D_{3}$.

Proof. Substituting the expression for $g_{11}^{(1)}$ in (3.14a) and integrating with respect to $d \tau$, we obtain

$$
\Phi_{11}^{(1)}(t, k)=\frac{i\left(e^{-4 i k^{2} t}-e^{i \omega t}\right)}{2 k+i \sqrt{\omega}}+\frac{1}{4} \int_{\partial \tilde{D}_{3}} \frac{e^{-4 i k_{3}^{2} t}-e^{-4 i k^{2} t}}{k^{2}-k_{3}^{2}} A\left(k_{3}^{2}\right) d k_{3} .
$$

The integrand in the rhs of (3.16) has removable singularities at $k_{3}=k$ and $k_{3}=-k$. In what follows it will be necessary to split the integral in (3.16) in two separate integrals. Thus, before splitting we deform the contour $\partial \tilde{D}_{3}$ to the contour $\partial \tilde{D}_{3}^{\sigma k}$ shown in figure 1 . One of these two integrals can be computed exactly via the residue theorem:

$$
\begin{aligned}
& -\frac{2 e^{-4 i k^{2} t}}{\pi} \int_{\partial \tilde{D}_{3}^{\sigma k}} \frac{k_{3}^{2}}{\left(4 k_{3}^{2}+\omega\right)\left(k^{2}-k_{3}^{2}\right)} d k_{3} \\
& \quad=-4 i e^{-4 i k^{2} t}\left[-\frac{\sigma k}{2\left(4 k^{2}+\omega\right)}+\left.\frac{k_{3}}{8\left(k^{2}-k_{3}^{2}\right)}\right|_{k_{3}=-i \sqrt{\omega} / 2}\right]=\frac{i e^{-4 i k^{2} t}}{2 \sigma k-i \sqrt{\omega}} .
\end{aligned}
$$

Substituting this expression in (3.16), where $\partial \tilde{D}_{3}$ is replaced with $\partial \tilde{D}_{3}^{\sigma k}$, we find (3.15).

For $k=0$, a separate analysis shows that the above integral equals $-1 / \sqrt{\omega}$, which agrees with the limit of the above expression as $k \rightarrow 0$. 
Lemma 3.2. For $\omega>0$,

$$
\Phi_{11}^{(2)}(t, k)=\frac{e^{-4 i k^{2} t}}{2 i \sigma k+\eta}+\frac{1}{i \pi} \int_{\partial \tilde{D}_{3}^{\sigma k}} \frac{k_{3} e^{-4 i k_{3}^{2} t}}{\left(2 i k_{3}+\eta\right)\left(k^{2}-k_{3}^{2}\right)} d k_{3} .
$$

Moreover,

$$
\overline{\Phi_{11}(t,-\bar{k})}=\overline{\Phi_{11}^{(1)}(t,-\bar{k})}-\frac{e^{4 i k^{2} t}}{2 i \hat{\sigma} k-\eta}+\frac{1}{i \pi} \int_{\partial \tilde{D}_{2}^{\sigma k}} \frac{k_{2} e^{4 i k_{2}^{2} t}}{\left(2 i k_{2}-\eta\right)\left(k^{2}-k_{2}^{2}\right)} d k_{2},
$$

where the contour $\partial \tilde{D}_{2}^{\hat{\sigma} k}$ is depicted in figure 2 and

$$
\overline{\Phi_{11}^{(1)}(t,-\bar{k})}=\frac{i\left(e^{4 i k^{2} t}-e^{-i \omega t}\right)}{2 k+i \sqrt{\omega}}-\frac{i e^{4 i k^{2} t}}{2 \hat{\sigma} k+i \sqrt{\omega}}+\frac{1}{4} \int_{\partial \tilde{D}_{2}^{\tilde{\sigma} k}} \frac{e^{4 i k_{2}^{2} t}}{k^{2}-k_{2}^{2}} A\left(k_{2}^{2}\right) d k_{2} .
$$

Proof. Substituting (3.12b) into (3.14b) and integrating the resulting expression with respect to $d \tau$, we find that $\Phi_{11}^{(2)}(t, k)$ is given by

$$
\Phi_{11}^{(2)}(t, k)=\frac{e^{-4 i k^{2} t}}{i \pi} \int_{\partial D_{3}} \frac{k_{3}}{2 i k_{3}+\eta}\left(\frac{e^{4 i t\left(k^{2}-k_{3}^{2}\right)}-1}{k^{2}-k_{3}^{2}}\right) d k_{3} .
$$

The integrand in (3.20) has removable singularities at $k_{3}= \pm k$. Thus, before splitting the integral we deform the contour $\partial D_{3}$ into $\partial \tilde{D}_{3}^{\sigma k}$. Note that it is not necessary to avoid $k=-i \sqrt{\omega} / 2$. Using the residue theorem, one of these integrals can be computed as

$$
-\frac{e^{-4 i k^{2} t}}{i \pi} \int_{\partial \tilde{D}_{3}^{\sigma k}} \frac{k_{3}}{\left(2 i k_{3}+\eta\right)\left(k^{2}-k_{3}^{2}\right)} d k_{3}=\frac{e^{-4 i k^{2} t}}{2 i \sigma k+\eta}
$$

and then we find $\Phi_{11}^{(2)}(t, k)$ given in (3.17).

Regarding $\overline{\Phi_{11}(t,-\bar{k})}$, we note that

$$
\overline{\Phi_{11}(t,-\bar{k})}=-e^{4 i k^{2} t} \int_{0}^{t} e^{-4 i k^{2} \tau}\left[2 k e^{-i \omega \tau}+i \bar{g}_{11}(\tau)\right] d \tau
$$

and $\bar{g}_{11}$ is given by

$$
\bar{g}_{11}(t)=\overline{g_{11}^{(1)}(t)}+\frac{4}{i \pi} \int_{\partial D_{2}} \frac{k_{2} e^{4 i k_{2}^{2} t}}{2 i k_{2}-\eta} d k_{2}
$$

with

$$
\overline{g_{11}^{(1)}(t)}=-\sqrt{\omega} e^{-i \omega t}+\int_{\partial \tilde{D}_{2}} e^{4 i k_{2}^{2} t} A\left(k_{2}^{2}\right) d k_{2},
$$

where $\partial D_{2}$ is the oriented boundary of the second quadrant of the complex $k$-plane and the contour $\partial \tilde{D}_{2}$ is depicted in figure 2. Substituting (3.22) and (3.23) into (3.21) and then integrating the resulting expression with respect to $d \tau$, we find

$$
\overline{\Phi_{11}(t,-\bar{k})}=\frac{i\left(e^{4 i k^{2} t}-e^{-i \omega t}\right)}{2 k+i \sqrt{\omega}}+\frac{1}{4} \int_{\tilde{D}_{2}} \frac{A\left(k_{2}^{2}\right)\left(e^{4 i k_{2}^{2} t}-e^{4 i k^{2} t}\right)}{k^{2}-k_{2}^{2}} d k_{2}+\frac{1}{i \pi} \int_{\partial D_{2}} \frac{k_{2}\left(e^{4 i k_{2}^{2} t}-e^{4 i k^{2} t}\right)}{\left(2 i k_{2}-\eta\right)\left(k^{2}-k_{2}^{2}\right)} d k_{2} .
$$

The integrands in the above expression have removable singularities at $k_{2}= \pm k$. Thus, before splitting the integrals we deform the contour $\partial D_{2}$ into $\partial \tilde{D}_{2}^{\tilde{\sigma} k}$, however it is not necessary to avoid $k=i \sqrt{\omega} / 2$, and then we find (3.18) by using the residue theorem. 
G. Hwang
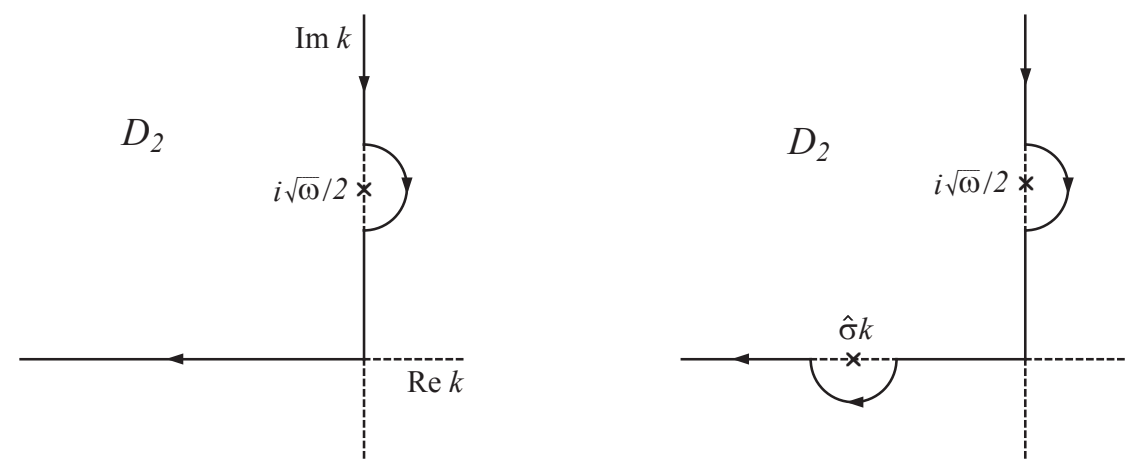

Fig. 2. $(\omega>0)$ The deformed contours $\partial \tilde{D}_{2}$ (left) and $\partial \tilde{D}_{2}^{\hat{\sigma} k}$ (right).

Let us denote $\lambda \Phi_{22}(t, k)$ by

$$
\lambda \Phi_{22}(t, k)=\lambda \Phi_{22}^{(1)}(t, k)+\lambda \Phi_{22}^{(2)}(t, k),
$$

where

and

$$
\lambda \Phi_{22}^{(1)}(t, k)=\int_{0}^{t}\left[2 k \bar{g}_{01}(\tau)-\overline{i g_{11}^{(1)}(\tau)}\right] \Phi_{11}^{(1)}(\tau, k) d \tau+i \int_{0}^{t}\left|g_{01}(\tau)\right|^{2} d \tau
$$

$$
\lambda \Phi_{22}^{(2)}(t, k)=-i \int_{0}^{t} \overline{g_{11}^{(2)}(\tau)} \Phi_{11}^{(1)}(\tau, k) d \tau+\int_{0}^{t}\left[2 k \bar{g}_{01}(\tau)-i \bar{g}_{11}(\tau)\right] \Phi_{11}^{(2)}(\tau, k) d \tau .
$$

Lemma 3.3. For $\omega>0$,

$$
\begin{aligned}
\lambda \Phi_{22}^{(1)}(t, k)= & \frac{i \alpha_{1}(k)}{2 k-i \sqrt{\omega}} e^{-i t\left(4 k^{2}+\omega\right)}+c_{1}(k)+\int_{\partial \tilde{D}_{3}^{\sigma k}} c_{2}\left(k, k_{3}\right) e^{-i t\left(4 k_{3}^{2}+\omega\right)} d k_{3} \\
& +\int_{\partial \tilde{D}_{2}^{\tilde{\sigma} k}} c_{3}\left(k, k_{2}\right) e^{4 i t\left(k_{2}^{2}-k^{2}\right)} d k_{2}+\int_{\partial \tilde{D}_{2}} c_{4}\left(k, k_{2}\right) e^{i t\left(4 k_{2}^{2}+\omega\right)} d k_{2} \\
& +\int_{\partial \tilde{D}_{3}^{\sigma k}}\left(\int_{\partial \tilde{D}_{2}^{\tilde{\sigma} k_{3}}} c_{5}\left(k, k_{2}, k_{3}\right) e^{4 i t\left(k_{2}^{2}-k_{3}^{2}\right)} d k_{2}\right) d k_{3},
\end{aligned}
$$

where the contours $\partial \tilde{D}_{2}$ and $\partial \tilde{D}_{2}^{\hat{\sigma} k}$ are depicted in figure $2, \hat{\sigma}=1$ if $k \in \partial D_{2}, \hat{\sigma}=-1$ if $-k \in \partial D_{2}$, and similarly for $\partial \tilde{D}_{2}^{\tilde{\sigma} k_{3}}$, with $\tilde{\sigma}=1$ if $k_{3} \in \partial D_{3}, \tilde{\sigma}=-1$ if $-k_{3} \in \partial D_{3}$. Furthermore, the functions $\alpha_{1}(k)$ and $\left\{c_{j}\right\}_{1}^{5}$ are defined as follows:

$$
\begin{array}{ll}
\alpha_{1}(k)=\frac{i}{2 k+i \sqrt{\omega}}+\frac{i}{2 \sigma k-i \sqrt{\omega}}, & c_{1}(k)=c_{1}^{(1)}(k)+c_{1}^{(2)}(k)+c_{1}^{(3)}(k), \\
c_{2}\left(k, k_{3}\right)=-\frac{1}{4 i\left(k^{2}-k_{3}^{2}\right)} \frac{A\left(k_{3}^{2}\right)}{2 k-i \sqrt{\omega}}, & c_{3}\left(k, k_{2}\right)=\frac{\alpha_{1}(k)}{4} \frac{A\left(k_{2}^{2}\right)}{\left(k^{2}-k_{2}^{2}\right)}, \\
c_{4}\left(k, k_{2}\right)=\frac{i A\left(k_{2}^{2}\right)}{(2 k+i \sqrt{\omega})\left(4 k_{2}^{2}+\omega\right)}, & c_{5}\left(k, k_{2}, k_{3}\right)=-\frac{1}{16} \frac{A\left(k_{2}^{2}\right) A\left(k_{3}^{2}\right)}{\left(k_{2}^{2}-k_{3}^{2}\right)\left(k^{2}-k_{3}^{2}\right)},
\end{array}
$$


where

$$
\begin{aligned}
c_{1}^{(1)}(k)= & \frac{2 k(\sigma+1)}{\left(4 k^{2}+\omega\right)(2 \sigma k-i \sqrt{\omega})}-\frac{1}{2 i \sqrt{\omega}} \frac{2 k+i \sqrt{\omega}}{(2 \sigma k-i \sqrt{\omega})^{2}}-\frac{1}{2 i \sqrt{\omega}(2 k+i \sqrt{\omega})}, \\
c_{1}^{(2)}(k)= & \frac{1}{2 \hat{\sigma} k+i \sqrt{\omega}}\left(\frac{1}{2 k+i \sqrt{\omega}}+\frac{1}{2 \sigma k-i \sqrt{\omega}}\right), \\
c_{1}^{(3)}(k)= & \frac{4 k^{2}}{i \pi\left(4 k^{2}+\omega\right)^{2}}(\log 4-\log \omega)+\frac{2 k \log k}{i \pi\left(4 k^{2}+\omega\right)(2 k+i \sqrt{\omega})} \\
& +\frac{2 k \log (-k)}{i \pi\left(4 k^{2}+\omega\right)(2 k-i \sqrt{\omega})}-\frac{1}{i \pi\left(4 k^{2}+\omega\right)}+\frac{4 k^{2}-\omega}{2\left(4 k^{2}+\omega\right)^{2}} .
\end{aligned}
$$

Proof. We note that

$$
-i \overline{g_{11}^{(1)}(\tau)}+2 k \bar{g}_{01}(\tau)=(2 k+i \sqrt{\omega}) e^{-i \omega t}-i \int_{\partial \tilde{D}_{2}} e^{4 i k_{2}^{2} \tau} A\left(k_{2}^{2}\right) d k_{2} .
$$

Let us denote $\Phi_{11}^{(1)}$ by

$$
\Phi_{11}^{(1)}=\alpha_{1}(k) e^{-4 i k^{2} t}+\alpha_{2}(k) e^{i \omega t}+\frac{1}{4} \int_{\partial \tilde{D}_{3}^{\sigma k}} \frac{e^{-4 i k_{3}^{2} t}}{k^{2}-k_{3}^{2}} A\left(k_{3}^{2}\right) d k_{3},
$$

with $\alpha_{1}(k)$ defined by the first equation in (3.27) and $\alpha_{2}(k)$ defined by

$$
\alpha_{2}(k)=-\frac{i}{2 k+i \sqrt{\omega}}
$$

Thus,

$$
\begin{aligned}
& \int_{0}^{t}\left(-i \overline{g_{11}^{(1)}(\tau)}+2 k \bar{g}_{01}(\tau)\right) \Phi_{11}^{(1)}(\tau, k) d \tau \\
& =(2 k+i \sqrt{\omega}) \int_{0}^{t} e^{-i \omega \tau}\left[\alpha_{1}(k) e^{-4 i k^{2} \tau}+\alpha_{2}(k) e^{i \omega \tau}+\frac{1}{4} \int_{\partial \tilde{D}_{3}^{\sigma k}} \frac{e^{-4 i k_{3}^{2} \tau} A\left(k_{3}^{2}\right)}{k^{2}-k_{3}^{2}} d k_{3}\right] d \tau \\
& -i \int_{0}^{t}\left\{\int_{\partial \tilde{D}_{2}} e^{4 i k_{2}^{2} \tau} A\left(k_{2}^{2}\right)\left[\alpha_{1}(k) e^{-4 i k^{2} \tau}+\alpha_{2}(k) e^{i \omega \tau}+\frac{1}{4} \int_{\partial \tilde{D}_{3}^{\sigma k}} \frac{e^{-4 i k_{3}^{2} \tau} A\left(k_{3}^{2}\right)}{k^{2}-k_{3}^{2}} d k_{3}\right] d k_{2}\right\} d \tau .
\end{aligned}
$$

Integrating with respect to $d \tau$, we find that the rhs of (3.33) equals the following expression:

$$
\begin{aligned}
(2 k & +i \sqrt{\omega})\left\{\alpha_{1}(k) \frac{e^{-i t\left(4 k^{2}+\omega\right)}-1}{-i\left(4 k^{2}+\omega\right)}+\alpha_{2}(k) t+\frac{1}{4} \int_{\partial \tilde{D}_{3}^{\sigma k}} \frac{e^{-i t\left(4 k_{3}^{2}+\omega\right)}-1}{-i\left(4 k_{3}^{2}+\omega\right)} \frac{A\left(k_{3}^{2}\right)}{k^{2}-k_{3}^{2}} d k_{3}\right\} \\
& -i \alpha_{1}(k) \int_{\partial \tilde{D}_{2}} \frac{e^{4 i t\left(k_{2}^{2}-k^{2}\right)}-1}{4 i\left(k_{2}^{2}-k^{2}\right)} A\left(k_{2}^{2}\right) d k_{2}-i \alpha_{2}(k) \int_{\partial \tilde{D}_{2}} \frac{e^{i t\left(4 k_{2}^{2}+\omega\right)}-1}{i\left(4 k_{2}^{2}+\omega\right)} A\left(k_{2}^{2}\right) d k_{2} \\
& -\frac{i}{4} \int_{\partial \tilde{D}_{2}}\left(\int_{\partial \tilde{D}_{3}^{\sigma k}} \frac{e^{4 i t\left(k_{2}^{2}-k_{3}^{2}\right)}-1}{4 i\left(k_{2}^{2}-k_{3}^{2}\right)} \frac{A\left(k_{2}^{2}\right) A\left(k_{3}^{2}\right)}{k^{2}-k_{3}^{2}} d k_{3}\right) d k_{2} .
\end{aligned}
$$

Note that

$$
(2 k+i \sqrt{\omega}) \alpha_{2}(k) t=-i t,
$$




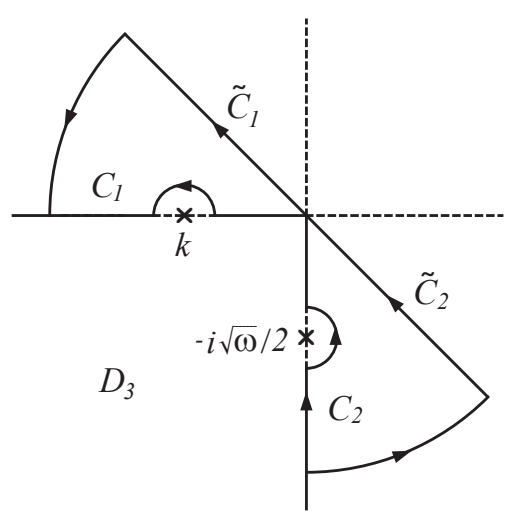

Fig. 3. The oriented contours $C_{1}, C_{2}, \tilde{C}_{1}$ and $\tilde{C}_{2}$.

which cancels the second integral of the rhs in (3.25a)

$$
i \int_{0}^{t}\left|g_{01}\right|^{2} d \tau=i t
$$

The single integrals involving $\partial \tilde{D}_{2}$ have removable singularities at $\pm k$; thus, before splitting these integrals we deform $\partial \tilde{D}_{2}$ to $\partial \tilde{D}_{2}^{\hat{\sigma} k}$.

Using the residue theorem, we find

$$
\begin{aligned}
& \int_{\partial \tilde{D}_{3}^{\sigma k}} \frac{A\left(k_{3}^{2}\right) d k_{3}}{\left(k^{2}-k_{3}^{2}\right)\left(4 k_{3}^{2}+\omega\right)}=-\frac{2}{\sqrt{\omega}(2 \sigma k-i \sqrt{\omega})^{2}}:=\beta_{1}(k), \\
& \int_{\partial \tilde{D}_{2}^{\hat{\sigma} k}} \frac{A\left(k_{2}^{2}\right) d k_{2}}{k^{2}-k_{2}^{2}}=\frac{4 i}{2 \hat{\sigma} k+i \sqrt{\omega}}:=\beta_{2}(k), \quad \int_{\partial \tilde{D}_{2}} \frac{A\left(k_{2}^{2}\right) d k_{2}}{4 k_{2}^{2}+\omega}=-\frac{1}{2 \sqrt{\omega}} .
\end{aligned}
$$

Regarding the double integral, we deform $\partial \tilde{D}_{2}$ to $\partial \tilde{D}_{2}^{\tilde{\sigma} k_{3}}$, where $\tilde{\sigma}=1$ if $k_{3} \in \partial D_{3}$ and $\tilde{\sigma}=-1$ if $-k_{3} \in \partial D_{3}$. Then, using a residue calculation similar to the one used in lemma 3.1, we find the identity

$$
\int_{\partial \tilde{D}_{2}^{\tilde{\sigma} k_{3}}} \frac{A\left(k_{2}^{2}\right)}{k_{2}^{2}-k_{3}^{2}} d k_{2}= \begin{cases}-\frac{4 i}{2 k_{3}+i \sqrt{\omega}} & \text { if } \quad \tilde{\sigma}=1, \\ \frac{4 i}{2 k_{3}-i \sqrt{\omega}} & \text { if } \quad \tilde{\sigma}=-1 .\end{cases}
$$

Thus, denoting the double integral with $\beta_{3}(k)$, we find

$$
\int_{\partial \tilde{D}_{3}^{\sigma k}}\left(\int_{\partial \tilde{D}_{2}^{\tilde{\sigma} k_{3}}} \frac{A\left(k_{2}^{2}\right) A\left(k_{3}^{2}\right)}{\left(k_{2}^{2}-k_{3}^{2}\right)\left(k^{2}-k_{3}^{2}\right)} d k_{2}\right) d k_{3}=\beta_{3}(k)=\frac{32}{i \pi}\left(\beta_{3}^{(1)}-\beta_{3}^{(2)}\right),
$$

where

$$
\begin{aligned}
& \beta_{3}^{(1)}(k)=\int_{C_{1}} \frac{k_{3}^{2}}{\left(4 k_{3}^{2}+\omega\right)} \frac{1}{\left(2 k_{3}+i \sqrt{\omega}\right)} \frac{1}{\left(k^{2}-k_{3}^{2}\right)} d k_{3}, \\
& \beta_{3}^{(2)}(k)=\int_{C_{2}} \frac{k_{3}^{2}}{\left(4 k_{3}^{2}+\omega\right)} \frac{1}{\left(2 k_{3}-i \sqrt{\omega}\right)} \frac{1}{\left(k^{2}-k_{3}^{2}\right)} d k_{3},
\end{aligned}
$$

and $C_{1}$ and $C_{2}$ denote the parts of $\partial \tilde{D}_{3}^{\sigma k}$ involving the negative real axis and the negative imaginary axis respectively (see figure 3 ). In order to compute $\beta_{3}^{(1)}$ and $\beta_{3}^{(2)}$, it is slightly more convenient 
to deform $C_{1}$ and $C_{2}$ to $\tilde{C}_{1}$ and $\tilde{C}_{2}$ respectively (see figure 3 ). Thus, using $k_{3}=r e^{3 i \pi / 4}=-r \alpha$, $\alpha=e^{-i \pi / 4}$ and $k_{3}=r \alpha$ for $\beta_{3}^{(1)}$ and $\beta_{3}^{(2)}$, respectively, we find

$$
\begin{aligned}
& \beta_{3}^{(1)}=e^{-i \pi / 4} \int_{0}^{\infty} \frac{i r^{2}}{\left(4 i r^{2}-\omega\right)} \frac{1}{\left(2 r e^{-i \pi / 4}-i \sqrt{\omega}\right)} \frac{1}{\left(k^{2}+i r^{2}\right)} d r, \\
& \beta_{3}^{(2)}=-\beta_{3}^{(1)} .
\end{aligned}
$$

Thus,

$$
\beta_{3}(k)=\frac{64}{i \pi} \beta_{3}^{(1)}(k)
$$

Computing $\beta_{3}^{(1)}(k)$ explicitly, we find

$$
\begin{aligned}
\beta_{3}(k)= & \frac{64 k^{2}}{i \pi\left(4 k^{2}+\omega\right)^{2}}(\log 4-\log \omega)+\frac{32 k \log k}{i \pi\left(4 k^{2}+\omega\right)(2 k+i \sqrt{\omega})} \\
& +\frac{32 k \log (-k)}{i \pi\left(4 k^{2}+\omega\right)(2 k-i \sqrt{\omega})}-\frac{16}{i \pi\left(4 k^{2}+\omega\right)}+\frac{8\left(4 k^{2}-\omega\right)}{\left(4 k^{2}+\omega\right)^{2}}
\end{aligned}
$$

Using the above formulas, we find (3.26), where $c_{1}(k)$ is defined by

$$
c_{1}(k)=\frac{(2 k+i \sqrt{\omega})}{i\left(4 k^{2}+\omega\right)} \alpha_{1}(k)+\frac{(2 k+i \sqrt{\omega})}{4 i} \beta_{1}(k)-\frac{\alpha_{1}(k)}{4} \beta_{2}(k)-\frac{\alpha_{2}(k)}{2 \sqrt{\omega}}+\frac{1}{16} \beta_{3}(k) .
$$

Simplifying the rhs of (3.34), we find that $c_{1}(k)$ is given by the second equation in (3.27) with (3.30).

Lemma 3.4. For $\omega>0$,

$$
\begin{aligned}
\lambda \Phi_{22}^{(2)}(t, k)= & \frac{i e^{-i t\left(4 k^{2}+\omega\right)}}{(2 i \sigma k+\eta)(2 k-i \sqrt{\omega})}+d_{1}(k)+\int_{\partial \tilde{D}_{3}^{\sigma k}} d_{2}\left(k, k_{3}\right) e^{-i t\left(4 k_{3}^{2}+\omega\right)} d k_{3} \\
& +\int_{\partial \tilde{D}_{2}^{\sigma k}} d_{3}\left(k, k_{2}\right) e^{i t\left(4 k_{2}^{2}+\omega\right)} d k_{2}+\int_{\partial \tilde{D}_{2}^{\tilde{\sigma} k}} d_{4}\left(k, k_{2}\right) e^{-4 i t\left(k^{2}-k_{2}^{2}\right)} d k_{2} \\
& +\int_{\partial \tilde{D}_{2}^{\tilde{\sigma} k}}\left(\int_{\partial \tilde{D}_{3}^{\sigma k}} d_{5}\left(k, k_{2}, k_{3}\right) e^{-4 i t\left(k_{2}^{2}-k_{3}^{2}\right)} d k_{3}\right) d k_{2},
\end{aligned}
$$

where the functions $\left\{d_{j}\right\}_{1}^{5}$ are defined by

$$
\begin{aligned}
d_{1}(k) & =d_{1}^{(1)}(k)+d_{1}^{(2)}(k)+d_{1}^{(3)}(k), \\
d_{2}\left(k, k_{3}\right) & =\frac{k_{3}}{\pi\left(2 i k_{3}+\eta\right)(2 k-i \sqrt{\omega})\left(k^{2}-k_{3}^{2}\right)}, \quad d_{3}\left(k, k_{2}\right)=\frac{4 k_{2}}{\pi(2 k+i \sqrt{\omega})\left(2 i k_{2}-\eta\right)\left(4 k_{2}^{2}+\omega\right)}, \\
d_{4}\left(k, k_{2}\right) & =\frac{k_{2}}{i \pi\left(2 i k_{2}-\eta\right)}\left(\frac{\alpha_{1}(k)}{2 i k_{2}-\eta}+\frac{1}{2 i \sigma k_{2}+\eta}\right)+\frac{A\left(k_{2}^{2}\right)}{4(2 i \sigma k+\eta)\left(k^{2}-k_{2}^{2}\right)},
\end{aligned}
$$

and 


$$
d_{5}\left(k, k_{2}, k_{3}\right)=\frac{1}{\pi\left(k^{2}-k_{3}^{2}\right)\left(k_{2}^{2}-k_{3}^{2}\right)}\left[\frac{k_{3}}{2 i k_{2}+\eta}\left(\frac{k_{2}}{\pi\left(2 i k_{3}-\eta\right)}-\frac{A\left(k_{2}^{2}\right)}{4 i}\right)-\frac{k_{2} A\left(k_{3}^{2}\right)}{4 i\left(2 i k_{2}-\eta\right)}\right]
$$

with

$$
\begin{aligned}
& d_{1}^{(1)}(k)=-\frac{i}{\eta+\sqrt{\omega}}\left(\frac{1}{2 k+i \sqrt{\omega}}+\frac{1}{2 k-i \sqrt{\omega}}\right), \\
& d_{1}^{(2)}(k)=-\frac{i}{2 i \hat{\sigma} k-\eta}\left(\frac{1}{2 k+i \sqrt{\omega}}+\frac{1}{2 \sigma k-i \sqrt{\omega}}\right)+\frac{i(\eta-\sqrt{\omega})}{(2 i \sigma k+\eta)(2 i \hat{\sigma} k-\eta)(2 \hat{\sigma} k+i \sqrt{\omega})},
\end{aligned}
$$

and

$$
\begin{aligned}
d_{1}^{(3)}( & k)=\frac{2 k^{2}(\pi+2 i)}{\pi\left(4 k^{2}+\eta^{2}\right)^{2}}-\frac{\eta^{2}(\pi-2 i)}{2 \pi\left(4 k^{2}+\eta^{2}\right)^{2}}-\frac{2 i k \log (-k)}{\pi(2 k+i \eta)(2 k-i \eta)^{2}}-\frac{2 i k \log k}{\pi(2 k-i \eta)(2 k+i \eta)^{2}} \\
- & \frac{8 i k^{2} \log (2 / \eta)}{\pi\left(4 k^{2}+\eta^{2}\right)^{2}}+\frac{2 i \omega \log \omega}{\pi\left(4 k^{2}+\omega\right)\left(\eta^{2}-\omega\right)}-\frac{4 \pi k^{2}-\pi \eta \sqrt{\omega}-16 i k^{2} \log 2}{\pi\left(4 k^{2}+\eta^{2}\right)\left(4 k^{2}+\omega\right)} \\
& -\frac{2 k(\eta+\sqrt{\omega})(\log (-k)-\log k)}{\pi\left(4 k^{2}+\eta^{2}\right)\left(4 k^{2}+\omega\right)}+\frac{8 i k^{2}(\log (-k)+\log k)}{\pi\left(4 k^{2}+\eta^{2}\right)\left(4 k^{2}+\omega\right)}-\frac{4 i \eta^{2} \log \eta}{\pi\left(4 k^{2}+\eta^{2}\right)\left(\eta^{2}-\omega\right)} .
\end{aligned}
$$

Proof. Recalling (3.12b), (3.14a) and (3.14b), as well as (3.1), we find that $\lambda \Phi_{22}^{(2)}(t, k)$ is given by

$$
\begin{aligned}
& \lambda \Phi_{22}^{(2)}(t, k)=\int_{0}^{t} 2 k e^{-i \omega \tau}\left[\frac{e^{-4 i k^{2} \tau}}{2 i \sigma k+\eta}+\frac{1}{i \pi} \int_{\partial \tilde{D}_{3}^{\sigma k}} \frac{k_{3} e^{-4 i k_{3}^{2} \tau}}{\left(2 i k_{3}+\eta\right)\left(k^{2}-k_{3}^{2}\right)} d k_{3}\right] d \tau \\
& -\frac{4}{\pi} \int_{0}^{t} \int_{\partial D_{2}} \frac{k_{2} e^{4 i k_{2}^{2} \tau}}{2 i k_{2}-\eta}\left[\Phi_{11}^{(1)}(\tau, k)+\frac{e^{-4 i k^{2} \tau}}{2 i \sigma k+\eta}+\frac{1}{i \pi} \int_{\partial \tilde{D}_{3}^{\sigma k}} \frac{k_{3} e^{-4 i k_{3}^{2} \tau}}{\left(2 i k_{3}+\eta\right)\left(k^{2}-k_{3}^{2}\right)} d k_{3}\right] d k_{2} d \tau \\
& -i \int_{0}^{t} \overline{g_{11}^{(1)}(\tau)}\left[\frac{e^{-4 i k^{2} \tau}}{2 i \sigma k+\eta}+\frac{1}{i \pi} \int_{\partial \tilde{D}_{3}^{\sigma k}} \frac{k_{3} e^{-4 i k_{3}^{2} \tau}}{\left(2 i k_{3}+\eta\right)\left(k^{2}-k_{3}^{2}\right)} d k_{3}\right] d \tau
\end{aligned}
$$

Integrating the first line in (3.38) with respect to $d \tau$, we find

$$
\frac{2 i k}{2 i \sigma k+\eta}\left(\frac{e^{-i t\left(4 k^{2}+\omega\right)}-1}{4 k^{2}+\omega}\right)+\frac{2 k}{\pi} \int_{\partial \tilde{D}_{3}^{\sigma k}} \frac{k_{3}\left(e^{-i t\left(4 k_{3}^{2}+\omega\right)}-1\right)}{\left(2 i k_{3}+\eta\right)\left(4 k_{3}^{2}+\omega\right)\left(k^{2}-k_{3}^{2}\right)} d k_{3} .
$$

Evaluating one of the integrals with respect to $d k_{3}$, (3.39) yields

$$
\frac{2 i k e^{-i t\left(4 k^{2}+\omega\right)}}{2 i \sigma k+\eta}-\frac{2 i k}{(\eta+\sqrt{\omega})\left(4 k^{2}+\omega\right)}+\frac{2 k}{\pi} \int_{\partial \tilde{D}_{3}^{\sigma k}} \frac{k_{3} e^{-i t\left(4 k_{3}^{2}+\omega\right)}}{\left(2 i k_{3}+\eta\right)\left(4 k_{3}^{2}+\omega\right)\left(k^{2}-k_{3}^{2}\right)} d k_{3},
$$

where we have used the identity

$$
\int_{\partial \tilde{D}_{3}^{\sigma k}} \frac{k_{3}}{\left(2 i k_{3}+\eta\right)\left(4 k_{3}^{2}+\omega\right)\left(k^{2}-k_{3}^{2}\right)} d k_{3}=-\frac{i \pi}{4 k^{2}+\omega}\left(\frac{1}{2 i \sigma k+\eta}-\frac{1}{\eta+\sqrt{\omega}}\right):=\beta_{4}(k) .
$$


Using (3.31) for $\Phi_{11}^{(1)}$ and then integrating the resulting expression with respect to $d \tau$, the second line in (3.38) becomes

$$
\begin{aligned}
& -\frac{4}{\pi} \int_{\partial D_{2}} \frac{k_{2}}{2 i k_{2}-\eta}\left\{\alpha_{1}(k) \frac{e^{-4 i t\left(k^{2}-k_{2}^{2}\right)}-1}{-4 i\left(k^{2}-k_{2}^{2}\right)}+\alpha_{2}(k) \frac{e^{i t\left(4 k_{2}^{2}+\omega\right)}-1}{i\left(4 k_{2}^{2}+\omega\right)}\right. \\
& +\frac{1}{4} \int_{\partial \tilde{D}_{3}^{\sigma k}} \frac{A\left(k_{3}^{2}\right)}{k^{2}-k_{3}^{2}}\left[\frac{e^{4 i t\left(k_{2}^{2}-k_{3}^{2}\right)}-1}{4 i\left(k_{2}^{2}-k_{3}^{2}\right)}\right] d k_{3}-\frac{e^{-4 i t\left(k^{2}-k_{2}^{2}\right)}-1}{4 i(2 i \sigma k+\eta)\left(k^{2}-k_{2}^{2}\right)} \\
& \left.\quad+\frac{i}{\pi} \int_{\partial \tilde{D}_{3}^{\sigma k}} \frac{k_{3}}{\left(2 i k_{3}+\eta\right)\left(k^{2}-k_{3}^{2}\right)}\left[\frac{e^{4 i t\left(k_{2}^{2}-k_{3}^{2}\right)}-1}{4 i\left(k_{2}^{2}-k_{3}^{2}\right)}\right] d k_{3}\right\} d k_{2} .
\end{aligned}
$$

Substituting (3.23) into (3.38) and integrating with respect to $d \tau$, the third line in (3.38) yields

$$
\begin{aligned}
- & \frac{\sqrt{\omega}}{2 i \sigma k+\eta}\left(\frac{e^{-i t\left(4 k^{2}+\omega\right)}-1}{4 k^{2}+\omega}\right)+\frac{\sqrt{\omega}}{\pi} \int_{\partial \tilde{D}_{3}^{\sigma k}} \frac{k_{3}}{\left(2 i k_{3}+\eta\right)\left(k^{2}-k_{3}^{2}\right)}\left[\frac{e^{-i t\left(4 k_{3}^{2}+\omega\right)}-1}{-i\left(4 k_{3}^{2}+\omega\right)}\right] d k_{3} \\
& -i \int_{\tilde{D}_{2}}\left\{\frac{A\left(k_{2}^{2}\right)}{2 i \sigma k+\eta}\left[\frac{e^{-4 i t\left(k^{2}-k_{2}^{2}\right)}-1}{-4 i\left(k^{2}-k_{2}^{2}\right)}\right]+\frac{A\left(k_{2}^{2}\right)}{i \pi} \int_{\partial \tilde{D}_{3}^{\sigma \sigma}} \frac{k_{3}}{\left(2 i k_{3}+\eta\right)\left(k-k_{3}^{2}\right)}\left[\frac{e^{4 i t\left(k_{2}^{2}-k_{3}^{2}\right)}-1}{4 i\left(k_{2}^{2}-k_{3}^{2}\right)}\right] d k_{3}\right\} d k_{2} .
\end{aligned}
$$

We can evaluate some of the integrals appearing in (3.41) and (3.42) explicitly. For the single integrals, from the residue theorem, we find

$$
\begin{aligned}
\int_{\partial \tilde{D}_{2}^{\sigma k}} \frac{k_{2}}{\left(2 i k_{2}-\eta\right)\left(k^{2}-k_{2}^{2}\right)} d k_{2} & =\frac{i \pi}{2 i \hat{\sigma} k-\eta} \\
\int_{\partial \tilde{D}_{2}^{\sigma k}} \frac{k_{2}}{\left(2 i k_{2}-\eta\right)\left(4 k_{2}^{2}+\omega\right)} d k_{2} & =\frac{i \pi}{4(\eta+\sqrt{\omega})} .
\end{aligned}
$$

Furthermore, we denote the double integrals in (3.41) and (3.42) by

$$
\begin{aligned}
& \int_{\partial \tilde{D}_{2}^{\tilde{\sigma} k_{3}}} \frac{k_{2}}{2 i k_{2}-\eta} \int_{\partial \tilde{D}_{3}^{\sigma k}} \frac{A\left(k_{3}^{2}\right)}{\left(k^{2}-k_{3}^{2}\right)\left(k_{2}^{2}-k_{3}^{2}\right)} d k_{3} d k_{2}:=\beta_{5}(k), \\
& \int_{\partial \tilde{D}_{2}^{\tilde{\sigma} k_{3}}} \frac{k_{2}}{2 i k_{2}-\eta} \int_{\partial \tilde{D}_{3}^{\sigma k}} \frac{k_{3}^{2}}{\left(2 i k_{3}+\eta\right)\left(k^{2}-k_{3}^{2}\right)\left(k_{2}^{2}-k_{3}^{2}\right)} d k_{3} d k_{2}:=\beta_{6}(k), \\
& \int_{\partial \tilde{D}_{2}^{\tilde{\sigma} k_{3}}} A\left(k_{2}^{2}\right) \int_{\partial \tilde{D}_{3}^{\sigma k}} \frac{k_{3}^{2}}{\left(2 i k_{3}+\eta\right)\left(k^{2}-k_{3}^{2}\right)\left(k_{2}^{2}-k_{3}^{2}\right)} d k_{3} d k_{2}:=\beta_{7}(k) .
\end{aligned}
$$

Thus equation (3.41) involves the following explicit terms

$$
-\frac{\alpha_{1}(k)}{2 i \hat{\sigma} k-\eta}+\frac{\alpha_{2}(k)}{\eta+\sqrt{\omega}}-\frac{1}{(2 i \sigma k+\eta)(2 i \hat{\sigma} k-\eta)}+\frac{\beta_{5}(k)}{4 i \pi}-\frac{\beta_{6}(k)}{\pi^{2}} .
$$

Similarly, for (3.42), we find

$$
-\frac{\sqrt{\omega}}{2 i \sigma k+\eta}\left(\frac{e^{-i t\left(4 k^{2}+\omega\right)}-1}{4 k^{2}+\omega}\right)+\frac{\sqrt{\omega}}{i \pi} \beta_{4}(k)-\frac{\beta_{2}(k)}{4(2 i \sigma k+\eta)}+\frac{\beta_{7}(k)}{4 i \pi} .
$$

The double integrals in (3.43) can be evaluated in a similar way as the term $\beta_{3}(k)$ in lemma 3.3; tedious but straightforward calculations yield

$$
\frac{1}{4 i \pi}\left(\beta_{5}(k)+\beta_{7}(k)\right)-\frac{\beta_{6}(k)}{\pi^{2}}=d_{1}^{(3)}(k),
$$


where $d_{1}^{(3)}$ is given in $(3.37 \mathrm{c})$.

Combining (3.40), (3.44) and (3.45), $\lambda \Phi_{22}^{(2)}(t, k)$ is given in (3.35) with (3.36).

Proposition 3.2. For $\omega>0$,

$$
\lambda g_{13}(t)=-\frac{1}{2 \sqrt{\omega}} e^{i \omega t}+o(1), \quad t \rightarrow \infty .
$$

Proof. First, note that (2.9) can be written in the form

$$
\lambda g_{13}(t)=\lambda g_{13}^{(1)}(t)+\lambda g_{13}^{(2)}(t)+\lambda g_{13}^{(3)}(t),
$$

where

$$
\begin{aligned}
& \lambda g_{13}^{(1)}(t)=\frac{2}{i \pi} \int_{\partial D_{3}} k\left[\lambda \Phi_{13}(t, k)-\lambda \Phi_{13}(t,-k)\right] d k, \\
& \lambda g_{13}^{(2)}(t)=\frac{2}{\pi} e^{i \omega t} \int_{\partial D_{3}}\left[\lambda \Phi_{22}(t, k)-\lambda \Phi_{22}(t,-k)\right] d k
\end{aligned}
$$

and

$$
\lambda g_{13}^{(3)}(t)=-\frac{4}{i \pi} \int_{\partial D_{3}} k e^{-4 i k^{2} t} b_{1}(-k)\left[\lambda \overline{\Phi_{22}(t,-\bar{k})}-\lambda a_{2}(-k)\right] d k .
$$

The expression for $\Phi_{22}(t, k)$ needed in (3.49) is given by (3.24) with (3.26) and (3.35) with $\sigma=1$; similarly $\Phi_{22}(t,-k)$ can be obtained by evaluating the rhs of (3.24) with (3.26) and (3.35) for $\sigma=-1$ and then replacing $k$ by $-k$. In what follows, we will determine the terms involving $e^{i \omega t}$ in (3.47).

In order to compute $\lambda g_{13}^{(3)}(t)$ involving $e^{i \omega t}$, we will first show that

$$
\frac{4}{i \pi} \int_{\partial D_{3}} k e^{-4 i k^{2} t} b_{1}(-k) \lambda a_{2}(-k) d k=O\left(t^{-3 / 2}\right), \quad t \rightarrow \infty .
$$

Recalling (2.14), we find

$$
\lambda a_{2}(-k)=\int_{0}^{\infty} e^{(2 i k-\eta) x} \int_{x}^{\infty} e^{-(2 i k+\eta) x^{\prime}} d x^{\prime} d x=\frac{1}{2 \eta(2 i k+\eta)} .
$$

Thus, the lhs of (3.51) with (3.4) yields

$$
-\frac{2}{i \eta \pi} \int_{\partial D_{3}} \frac{k e^{-4 i k^{2} t}}{(2 i k+\eta)^{2}} d k
$$

Using a similar analysis as in proposition 3.1, we can show that (3.52) is of $O\left(t^{-3 / 2}\right)$ as $t \rightarrow \infty$. We next consider the following integral

$$
-\frac{4}{i \pi} \int_{\partial D_{3}} k e^{-4 i k^{2} t} b_{1}(-k) \lambda \overline{\Phi_{22}(t,-\bar{k})} d k
$$

where from (2.11), $\lambda \overline{\Phi_{22}(t,-\bar{k})}$ is given by

$$
\lambda \overline{\Phi_{22}(t,-\bar{k})}=\int_{0}^{t}\left[-2 k e^{i \omega \tau}+i g_{11}(\tau)\right] \overline{\Phi_{11}(\tau,-\bar{k})} d k-i \int_{0}^{t}\left|g_{01}\right|^{2} d \tau .
$$


From (3.18), we write $\overline{\Phi_{11}(t,-\bar{k})}$ as

$$
\begin{aligned}
\overline{\Phi_{11}(t,-\bar{k})}= & \tilde{\alpha}_{1}(k) e^{4 i k^{2} t}+\alpha_{2}(k) e^{i \omega t}+\frac{1}{4} \int_{\partial \tilde{D}_{2}^{\sigma k}} \frac{A\left(k_{2}^{2}\right) e^{4 i k_{2}^{2} t}}{k^{2}-k_{2}^{2}} d k_{2} \\
& +\frac{1}{i \pi} \int_{\partial \tilde{D}_{2}^{\sigma k}} \frac{k_{2} e^{4 i k_{2}^{2} t}}{\left(2 i k_{2}-\eta\right)\left(k^{2}-k_{2}^{2}\right)} d k_{2},
\end{aligned}
$$

where $\alpha_{2}(k)$ is given in (3.32) and

$$
\tilde{\alpha}_{1}(k)=\frac{i}{2 k+i \sqrt{\omega}}-\frac{i}{2 \hat{\sigma} k+i \sqrt{\omega}}-\frac{1}{2 \hat{\sigma} k-\eta} .
$$

Substituting (3.55) into (3.54) and using a similar calculation as in lemma 3.3, the term involving $e^{i \omega t}$ in (3.54) arises from

$$
\frac{i \tilde{\alpha}_{1}(k)}{2 k-i \sqrt{\omega}}
$$

and (3.53) implies that the relevant contribution is

$$
\begin{aligned}
\frac{4}{\pi} \int_{\partial \tilde{D}_{3}} \frac{k \tilde{\alpha}_{1}(k)}{(2 k-i \sqrt{\omega})(2 i k+\eta)} d k & =-\frac{4}{\pi}\left\{\int_{C_{1}} \frac{k}{(2 i k-\eta)(2 i k+\eta)(2 k-i \sqrt{\omega})}\right. \\
& \left.-\int_{C_{2}} \frac{k}{(2 i k+\eta)(2 k-i \sqrt{\omega})}\left(\frac{4 i k}{4 k^{2}+\omega}+\frac{1}{2 i k+\eta}\right) d k\right\} .
\end{aligned}
$$

Deforming each contour into $\tilde{C}_{1}$ and $\tilde{C}_{2}$ and letting $k=-r \alpha$ and $k=r \alpha$, respectively, we can evaluate the rhs of the above equation and we obtain

$$
\lambda g_{13}^{(3)}(t)=\left(-\frac{1}{\eta+\sqrt{\omega}}-\frac{2}{i \pi(\eta-\sqrt{\omega})}+\frac{\left(\eta^{2}+\omega\right) \log \left(\eta^{2} / \omega\right)}{i \pi(\eta-\sqrt{\omega})\left(\eta^{2}-\omega\right)}\right) e^{i \omega t}+o(1), \quad t \rightarrow \infty .
$$

We next compute the term in $\lambda g_{13}^{(2)}(t)$ involving $e^{i \omega t}$. Recalling (3.13), the term involving $e^{i \omega t}$ arises from

$$
\frac{2}{\pi} \int_{\partial \tilde{D}_{3}}\left[c_{1}(k)-c_{1}(-k)\right] d k+\frac{2}{\pi} \int_{\partial \tilde{D}_{3}}\left[d_{1}(k)-d_{1}(-k)\right] d k .
$$

Note that $c_{1}(k)=c_{1}^{(1)}(k)+c_{1}^{(2)}(k)+c_{1}^{(3)}(k)$ with $c_{1}^{(2)}(-k)=0$ and $c_{1}^{(3)}(k)=c_{1}^{(3)}(-k)$. Hence, for the first term in (3.58) we need to evaluate the integral

$$
\frac{2}{\pi} \int_{\partial \tilde{D}_{3}}\left[c_{1}^{(1)}(k)-c_{1}^{(1)}(-k)\right] d k+\frac{2}{\pi} \int_{\partial \tilde{D}_{3}} c_{1}^{(2)}(k) d k .
$$

For the first integral in (3.59), we find

$$
c_{1}^{(1)}(k)-c_{1}^{(1)}(-k)=\frac{4 i k}{\sqrt{\omega}\left(4 k^{2}+\omega\right)},
$$

which has simple poles at $k=-i \sqrt{\omega} / 2$ and $k=\infty$. Hence, the first integral in (3.59) is given by

$$
\frac{2}{\pi} \int_{\partial \tilde{D}_{3}}\left[c_{1}^{(1)}(k)-c_{1}^{(1)}(-k)\right] d k=-\frac{1}{\sqrt{\omega}} .
$$

In order to compute the second integral in (3.59), we introduce as before $C_{1}$ and $C_{2}$ as the parts of $\partial \tilde{D}_{3}$ involving the negative real axis and the negative imaginary axis respectively (cf. figure 3 ). 
Thus, the second integral in (3.59) can be written in the form

$$
\frac{2}{\pi} \int_{\partial \tilde{D}_{3}} c_{1}^{(2)}(k) d k=\frac{8}{\pi}\left(\tilde{c}_{1}^{(1)}-\tilde{c}_{1}^{(2)}\right),
$$

where

$$
\tilde{c}_{1}^{(1)}=\int_{C_{1}} \frac{k}{(2 k+i \sqrt{\omega})^{2}(2 k-i \sqrt{\omega})} d k, \quad \tilde{c}_{1}^{(2)}=\int_{C_{2}} \frac{k}{(2 k+i \sqrt{\omega})(2 k-i \sqrt{\omega})^{2}} d k .
$$

In order to evaluate the above integrals, we deform $C_{1}$ and $C_{2}$ to $\tilde{C}_{1}$ and $\tilde{C}_{2}$, respectively and then use $k_{3}=-r \alpha, \alpha=e^{-i \pi / 4}$ and $k_{3}=r \alpha$ for $\tilde{c}_{1}^{(1)}$ and $\tilde{c}_{1}^{(2)}$, respectively (cf. figure 3); this yields $\tilde{c}_{1}^{(1)}=\tilde{c}_{1}^{(2)}$ and hence,

$$
\frac{2}{\pi} \int_{\partial \tilde{D}_{3}} c_{1}^{(2)}(k) d k=0
$$

Thus, we find

$$
\frac{2}{\pi} \int_{\partial \tilde{D}_{3}}\left[c_{1}(k)-c_{1}(-k)\right] d k=-\frac{1}{\sqrt{\omega}}
$$

For the second term in (3.58), we note that $d_{1}^{(3)}(k)=d_{1}^{(3)}(-k)$, and hence we need to compute

$$
\frac{2}{\pi} \int_{\partial \tilde{D}_{3}}\left[d_{1}^{(1)}(k)-d_{1}^{(1)}(-k)\right] d k+\frac{2}{\pi} \int_{\partial \tilde{D}_{3}}\left[d_{1}^{(2)}(k)-d_{1}^{(2)}(-k)\right] d k .
$$

The integrand of the first integral in (3.62) can be simplified to

$$
d_{1}^{(1)}(k)-d_{1}^{(1)}(-k)=-\frac{8 i k}{(\eta+\sqrt{\omega})\left(4 k^{2}+\omega\right)},
$$

and then we find

$$
\frac{2}{\pi} \int_{\partial \tilde{D}_{3}}\left[d_{1}^{(1)}(k)-d_{1}^{(1)}(-k)\right] d k=\frac{2}{\eta+\sqrt{\omega}}
$$

where we have used the fact that the integrand has poles at $k=-i \sqrt{\omega} / 2$ and at $k=\infty$. The second integral in (3.62) can be written as

$$
\frac{2}{\pi} \int_{\partial \tilde{D}_{3}}\left[d_{1}^{(2)}(k)-d_{1}^{(2)}(-k)\right] d k=\frac{8}{i \pi}\left\{\int_{C_{1}} \frac{k}{(2 i k-\eta)\left(4 k^{2}+\omega\right)} d k-\int_{C_{2}} \frac{k}{(2 i k+\eta)\left(4 k^{2}+\omega\right)} d k\right\} .
$$

Deforming each contour into $\tilde{C}_{1}$ and $\tilde{C}_{2}$ and letting $k=-r \alpha$ and $k=r \alpha$, respectively, we find that the integral in the rhs of the above equation is identically zero. Thus, from (3.63), we find

$$
\frac{2}{\pi} \int_{\partial \tilde{D}_{3}}\left[d_{1}(k)-d_{1}(-k)\right] d k=\frac{2}{\eta+\sqrt{\omega}} .
$$

Therefore, combining (3.64) with (3.61), the coefficient of the term $e^{i \omega t}$ for $\lambda g_{13}^{(2)}(t)$ is given by

$$
\lambda g_{13}^{(2)}(t)=\left(-\frac{1}{\sqrt{\omega}}+\frac{2}{\eta+\sqrt{\omega}}\right) e^{i \omega t}+o(1), \quad t \rightarrow \infty .
$$


In order to compute the analogous contributions from $\lambda g_{13}^{(1)}(t)$, we note that according to (2.12), $\lambda \Phi_{13}(t, k)$ involves two contributions. Noting that

$$
\begin{aligned}
& \int_{0}^{t}-i\left|g_{01}\right|^{2} e^{4 i k^{2} \tau} \Phi_{11}(\tau, k) d \tau=-i \int_{0}^{t} e^{4 i k^{2} \tau}\left[\alpha_{1}(k) e^{-4 i k^{2} \tau}+\alpha_{2}(k) e^{i \omega \tau}\right. \\
& \left.\quad+\frac{1}{4} \int_{\partial \tilde{D}_{3}^{\sigma k}} \frac{e^{-4 i k_{3}^{2} \tau}}{k^{2}-k_{3}^{2}} A\left(k_{3}^{2}\right) d k_{3}+\frac{e^{-4 i k^{2} t}}{2 i \sigma k+\eta}+\frac{1}{i \pi} \int_{\partial \tilde{D}_{3}^{\sigma k}} \frac{k_{3} e^{-4 i k_{3}^{2} t}}{\left(2 i k_{3}+\eta\right)\left(k^{2}-k_{3}^{2}\right)} d k_{3}\right] d \tau
\end{aligned}
$$

it follows that the $\lambda g_{13}^{(1)}(t)$ involves the following explicit terms:

$$
I_{1}(t, k)=-i e^{-4 i k^{2} t}\left\{\alpha_{1}(k) t+\frac{t}{2 i \sigma k+\eta}+\alpha_{2}(k) \frac{e^{i t\left(4 k^{2}+\omega\right)}-1}{i\left(4 k^{2}+\omega\right)}\right\} .
$$

Furthermore, noting that

$$
\begin{aligned}
& \int_{0}^{t}\left[2 k g_{01}(\tau)+i g_{11}(\tau)\right] e^{4 i k^{2} \tau} \lambda \Phi_{22}(\tau, k) d \tau \\
& =\int_{0}^{t} e^{4 i k^{2} \tau}\left[2 k e^{i \omega \tau}-i \sqrt{\omega} e^{i \omega \tau}+i \int_{\partial \tilde{D}_{3}} e^{-4 i k_{3}^{2} \tau} A\left(k_{3}^{2}\right) d k_{3}+\frac{4}{i \pi} \int_{\partial D_{3}} \frac{k e^{-4 i k^{2} t}}{2 i k+\eta} d k\right] \lambda \Phi_{22}(\tau, k) d \tau
\end{aligned}
$$

it follows that $\lambda g_{13}^{(1)}(t)$ also involves the following explicit terms:

$$
I_{2}(t, k)=e^{-4 i k^{2} t}\left\{i \alpha_{1}(k) t+\frac{i t}{2 i \sigma k+\eta}+\left(c_{1}(k)+d_{1}(k)\right) \frac{e^{i t\left(4 k^{2}+\omega\right)}-1}{i(2 k+i \sqrt{\omega})}\right\} .
$$

The expressions $I_{1}$ and $I_{2}$ yield the following asymptotic contributions:

$$
\begin{aligned}
\lambda g_{13}^{(1)}(t) \sim & \frac{2}{i \pi} \int_{\partial D_{3}} k\left[\left.I_{1}(t, k)\right|_{\sigma=1}-\left.I_{1}(t,-k)\right|_{\sigma=-1}\right] d k \\
& +\frac{2}{i \pi} \int_{\partial D_{3}} k\left[\left.I_{2}(t, k)\right|_{\sigma=1}-\left.I_{2}(t,-k)\right|_{\sigma=-1}\right] d k
\end{aligned}
$$

We next compute the contribution in (3.68) of terms involving $e^{i \omega t}$. These contributions arise from the third term in $I_{1}$ and from the third term in $I_{2}$.

The coefficient of $e^{i \omega t}$ from the third term of $I_{1}$ leads to

$$
-\frac{\alpha_{2}(k)}{4 k^{2}+\omega}=\frac{i}{(2 k+i \sqrt{\omega})\left(4 k^{2}+\omega\right)}
$$

and the relevant contribution in the first term of (3.68) equals

$$
\frac{2}{i \pi} \int_{\partial \tilde{D}_{3}} \frac{k}{4 k^{2}+\omega}\left[\frac{i}{2 k+i \sqrt{\omega}}+\frac{i}{2 k-i \sqrt{\omega}}\right] d k=-\frac{1}{2 \sqrt{\omega}}
$$

where we have used the fact that the above integrand has a double poles at $k=-i \sqrt{\omega} / 2$. 
The coefficient of $e^{i \omega t}$ from the third term in $I_{2}$ is given by

$$
\frac{c_{1}(k)+d_{1}(k)}{i(2 k+i \sqrt{\omega})} .
$$

Thus, the relevant contribution equals

$$
\frac{2}{i \pi} \int_{\partial \tilde{D}_{3}} k\left[\frac{c_{1}(k)+d_{1}(k)}{i(2 k+i \sqrt{\omega})}+\frac{c_{1}(-k)+d_{1}(-k)}{i(2 k-i \sqrt{\omega})}\right] d k .
$$

Recall that

$$
c_{1}(k)=c_{1}^{(1)}(k)+c_{1}^{(2)}(k)+c_{1}^{(3)}(k), \quad d_{1}(k)=d_{1}^{(1)}(k)+d_{1}^{(2)}(k)+d_{1}^{(3)}(k) .
$$

For $c_{1}^{(1)}(k)$, we find

$$
\frac{k c_{1}^{(1)}(k)}{2 k+i \sqrt{\omega}}+\frac{k c_{1}^{(1)}(-k)}{2 k-i \sqrt{\omega}}=\frac{8 k^{2}}{\left(4 k^{2}+\omega\right)^{2}},
$$

which has a pole at $k=-i \sqrt{\omega} / 2$, and hence

$$
-\frac{2}{\pi} \int_{\partial \tilde{D}_{3}} k\left[\frac{c_{1}^{(1)}(k)}{2 k+i \sqrt{\omega}}+\frac{c_{1}^{(1)}(-k)}{2 k-i \sqrt{\omega}}\right] d k=\frac{1}{\sqrt{\omega}} .
$$

For $c_{1}^{(2)}(k)$, we obtain

$$
-\frac{2}{\pi} \int_{\partial \tilde{D}_{3}} \frac{k c_{1}^{(2)}(k)}{2 k+i \sqrt{\omega}} d k=-\frac{8}{\pi} \int_{C_{1}} \frac{k^{2}}{\left(4 k^{2}+\omega\right)(2 k+i \sqrt{\omega})^{2}} d k+\frac{8}{\pi} \int_{C_{2}} \frac{k^{2}}{\left(4 k^{2}+\omega\right)^{2}} d k .
$$

The integrals in the rhs of (3.72) can be computed in a similar way as before; we find

$$
-\frac{2}{\pi} \int_{\partial \tilde{D}_{3}} \frac{k c_{1}^{(2)}(k)}{2 k+i \sqrt{\omega}} d k=-\frac{\pi-4 i}{8 \pi \sqrt{\omega}}
$$

For the term involving $c_{1}^{(3)}$, using $c_{1}^{(3)}(k)=c_{1}^{(3)}(-k)$, we find

$$
-\frac{2}{\pi} \int_{\partial \tilde{D}_{3}} k\left[\frac{1}{2 k+i \sqrt{\omega}}+\frac{1}{2 k-i \sqrt{\omega}}\right] c_{1}^{(3)}(k) d k=\frac{\pi-4 i}{8 \pi \sqrt{\omega}},
$$

where we have used the fact that the integrand has a pole at $k=-i \sqrt{\omega} / 2$. Combining (3.61), (3.69), (3.71), (3.73) and (3.74), we obtain the coefficient of $e^{i \omega t}$ as

$$
\frac{2}{i \pi} \int_{\partial \tilde{D}_{3}} k\left[\frac{c_{1}(k)}{i(2 k+i \sqrt{\omega})}+\frac{c_{1}(-k)}{i(2 k-i \sqrt{\omega})}\right] d k=\frac{1}{\sqrt{\omega}} .
$$

We will evaluate the integrals involving the term $d_{1}(k)$ in (3.70). First, noting that

$$
k\left[\frac{d_{1}^{(1)}(k)}{2 k+i \sqrt{\omega}}+\frac{d_{1}^{(1)}(-k)}{2 k-i \sqrt{\omega}}\right]=-\frac{8 \sqrt{\omega}}{(\eta+\sqrt{\omega})} \frac{k^{2}}{\left(4 k^{2}+\omega\right)^{2}},
$$

we find

$$
-\frac{2}{\pi} \int_{\partial \tilde{D}_{3}} k\left[\frac{d_{1}^{(1)}(k)}{2 k+i \sqrt{\omega}}+\frac{d_{1}^{(1)}(-k)}{2 k-i \sqrt{\omega}}\right] d k=-\frac{1}{\eta+\sqrt{\omega}},
$$

where we have used the fact that the integrand has a double pole at $k=-i \sqrt{\omega} / 2$. 
For $d_{1}^{(2)}$, we note that

$$
\begin{aligned}
&- \frac{2}{\pi} \int_{\partial \tilde{D}_{3}} k\left[\frac{d_{1}^{(2)}(k)}{2 k+i \sqrt{\omega}}+\frac{d_{1}^{(2)}(-k)}{2 k-i \sqrt{\omega}}\right] d k \\
&= \frac{8 i}{\pi}\left\{\int_{C_{1}} \frac{k^{2}}{(2 k+i \sqrt{\omega})(2 i k-\eta)\left(4 k^{2}+\omega\right)} d k-\int_{C_{2}} \frac{k^{2}}{(2 k+i \sqrt{\omega})(2 i k+\eta)\left(4 k^{2}+\omega\right)} d k\right\} \\
& \quad+\frac{8(\eta-\sqrt{\omega})}{i \pi}\left\{\int_{C_{1}} \frac{k^{2}}{(2 i k-\eta)(2 i k+\eta)(2 k+i \sqrt{\omega})\left(4 k^{2}+\omega\right)} d k\right. \\
&\left.\quad+\int_{C_{2}} \frac{k^{2}}{(2 i k+\eta)^{2}(2 k-i \sqrt{\omega})\left(4 k^{2}+\omega\right)} d k\right\} .
\end{aligned}
$$

As before, deforming each contours into $\tilde{C}_{1}$ and $\tilde{C}_{2}$ and letting $k=-r \alpha$ and $k=r \alpha$, (3.76) yields

$$
\begin{aligned}
-\frac{2}{\pi} & \int_{\partial \tilde{D}_{3}} k\left[\frac{d_{1}^{(2)}(k)}{2 k+i \sqrt{\omega}}+\frac{d_{1}^{(2)}(-k)}{2 k-i \sqrt{\omega}}\right] d k \\
& =\frac{\eta}{2(\eta+\sqrt{\omega})^{2}}+\frac{2 \eta-\sqrt{\omega}}{i \pi\left(\eta^{2}-\omega\right)}-\frac{\eta\left(\eta^{2}-\eta \sqrt{\omega}+\omega\right)}{i \pi\left(\eta^{2}-\omega\right)^{2}} \log \left(\eta^{2} / \omega\right) .
\end{aligned}
$$

Regarding $d_{1}^{(3)}$, using $d_{1}^{(3)}(k)=d_{1}^{(3)}(-k)$, we find

$$
\begin{aligned}
-\frac{2}{\pi} & \int_{\partial \tilde{D}_{3}} k\left[\frac{1}{2 k+i \sqrt{\omega}}+\frac{1}{2 k-i \sqrt{\omega}}\right] d_{1}^{(3)}(k) d k \\
& =-\frac{\eta}{2(\eta+\sqrt{\omega})^{2}}-\frac{3 i \sqrt{\omega}}{\pi\left(\eta^{2}-\omega\right)}-\frac{\sqrt{\omega}\left(2 \eta^{2}+\omega\right)}{i \pi\left(\eta^{2}-\omega\right)^{2}} \log \left(\eta^{2} / \omega\right)
\end{aligned}
$$

where we have used the fact that the integrand has poles at $k=-i \sqrt{\omega} / 2$ and at $k=-i \eta / 2$. Thus, combining (3.75), (3.77) and (3.78), we obtain

$$
\begin{aligned}
& \frac{2}{i \pi} \int_{\partial \tilde{D}_{3}} k\left[\frac{d_{1}(k)}{i(2 k+i \sqrt{\omega})}+\frac{d_{1}(-k)}{i(2 k-i \sqrt{\omega})}\right] d k \\
& \quad=-\frac{1}{\eta+\sqrt{\omega}}+\frac{2}{i \pi(\eta-\sqrt{\omega})}-\frac{\left(\eta^{2}+\omega\right) \log \left(\eta^{2} / \omega\right)}{i \pi(\eta-\sqrt{\omega})\left(\eta^{2}-\omega\right)}
\end{aligned}
$$

and then we find

$$
\begin{aligned}
\lambda g_{13}^{(1)}(t)= & -\frac{1}{2 \sqrt{\omega}}+\frac{1}{\sqrt{\omega}}-\frac{1}{\eta+\sqrt{\omega}}+\frac{2}{i \pi(\eta-\sqrt{\omega})} \\
& \left.-\frac{\left(\eta^{2}+\omega\right) \log \left(\eta^{2} / \omega\right)}{i \pi(\eta-\sqrt{\omega})\left(\eta^{2}-\omega\right)}\right) e^{i \omega t}+o(1), \quad t \rightarrow \infty .
\end{aligned}
$$

Therefore, combining equations (3.57), (3.65) and (3.79), we find (3.46).

The computations of the large $t$ asymptotics of the terms involving single integrals with respect to $d k_{2}$ and $d k_{3}$ of $e^{4 i k_{2}^{2} t}$ and $e^{4 i k_{3}^{2} t}$ respectively, as well as double integrals with respect to $d k_{2} d k_{3}$ of $e^{4 i\left(k_{2}^{2}-k_{3}^{2}\right) t}$, are similar with the computations presented in proposition 3.1. 

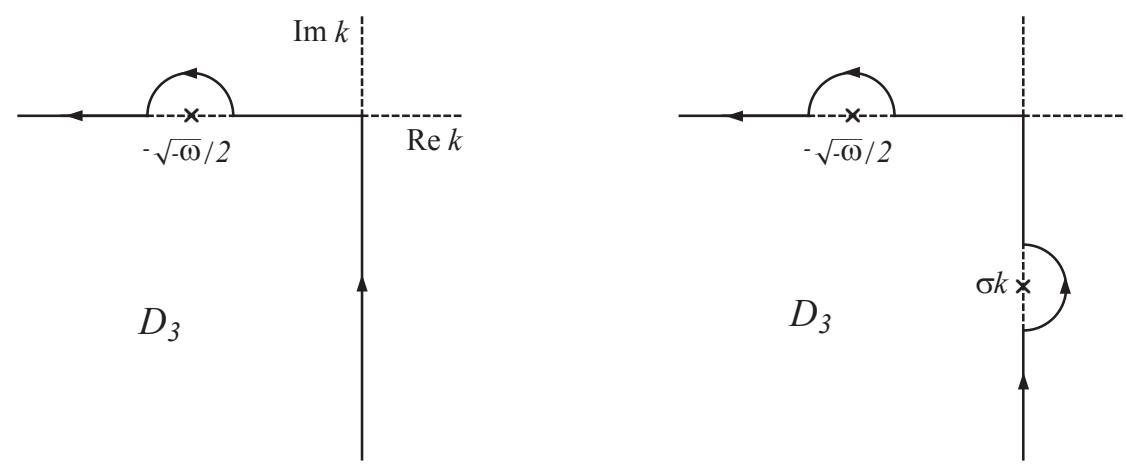

Fig. 4. $(\omega<0)$ The deformed contours $\partial \check{D}_{3}$ (left) and $\partial \check{D}_{3}^{\sigma k}$ (right).

\section{A Perturbative Approach for $\omega<0$}

In a similar way as in Section 3, we can obtain the analogous results for the case of $\omega<0$. For simplicity, here we present the results with a vanishing initial datum, that is, $q(x, 0)=0$. In this case, $a(k)=1$ and $b(k)=0$.

Proposition 4.1. For $\omega<0$,

$$
g_{11}(t)=i \sqrt{-\omega} e^{i \omega t}+\int_{\partial \check{D}_{3}} e^{-4 i k_{3}^{2} t} A\left(k_{3}^{2}\right) d k_{3},
$$

where the contour $\partial \check{D}_{3}$ is depicted in figure 4 and

$$
A\left(k^{2}\right)=\frac{8 k^{2}}{\pi\left(4 k^{2}+\omega\right)} .
$$

Lemma 4.1. For $\omega<0$,

$$
\Phi_{11}(t, k)=\frac{i\left(e^{-4 i k^{2} t}-e^{i \omega t}\right)}{2 k+\sqrt{-\omega}}+\frac{i e^{-4 i k^{2} t}}{2 \sigma k-\sqrt{-\omega}}+\frac{1}{4} \int_{\partial \check{D}_{3}^{\sigma k}} \frac{e^{-4 i k_{3}^{2} t}}{k^{2}-k_{3}^{2}} A\left(k_{3}^{2}\right) d k_{3},
$$

where the contour $\partial \check{D}_{3}^{\sigma k}$ is depicted in figure 4 and $\sigma=1$ if $k \in \partial D_{3}$, whereas $\sigma=-1$ if $-k \in \partial D_{3}$.

Lemma 4.2. For $\omega<0$,

$$
\begin{aligned}
\lambda \Phi_{22}(t, k)= & \frac{i \alpha_{1}(k)}{2 k+\sqrt{-\omega}} e^{-i t\left(4 k^{2}+\omega\right)}+\frac{2 i \sqrt{-\omega} t}{2 k+\sqrt{-\omega}}+c_{1}(k)+\int_{\partial \check{D}_{3}^{\sigma k}} c_{2}\left(k, k_{3}\right) e^{-i t\left(4 k_{3}^{2}+\omega\right)} d k_{3} \\
& +\int_{\partial \check{D}_{2}^{\sigma k}} c_{3}\left(k, k_{2}\right) e^{4 i t\left(k_{2}^{2}-k^{2}\right)} d k_{2}+\int_{\partial \check{D}_{2}} c_{4}\left(k, k_{2}\right) e^{i t\left(4 k_{2}^{2}+\omega\right)} d k_{2} \\
& +\int_{\partial \check{D}_{3}^{\sigma k}}\left(\int_{\partial \check{D}_{2}^{\tilde{\sigma} k_{3}}} c_{5}\left(k, k_{2}, k_{3}\right) e^{4 i t\left(k_{2}^{2}-k_{3}^{2}\right)} d k_{2}\right) d k_{3},
\end{aligned}
$$


A Perturbative Approach for the Asymptotic Evaluation of Neumann Value for the NLS
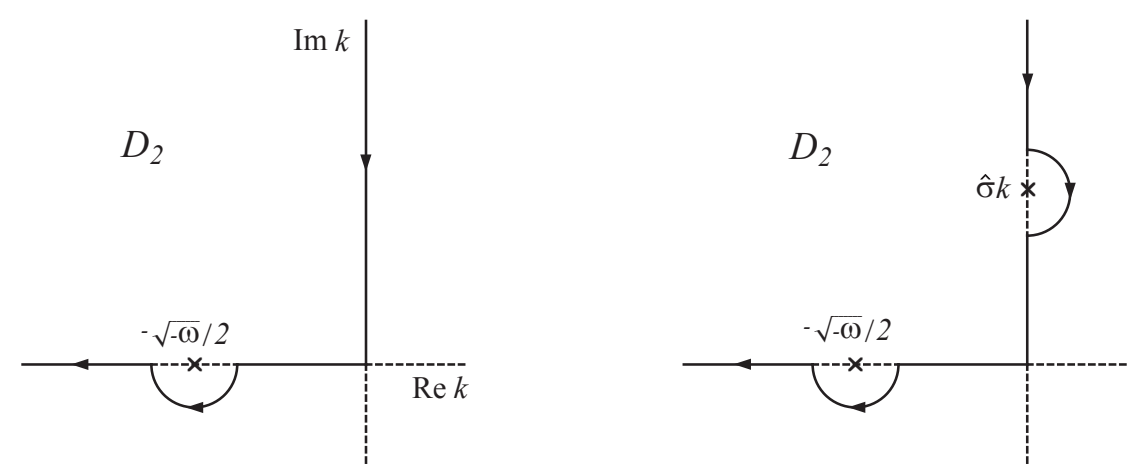

Fig. 5. $(\omega<0)$ The deformed contours $\partial \check{D}_{2}$ (left) and $\partial \check{D}_{2}^{\hat{\sigma} k}$ (right).

where the contours $\partial \check{D}_{2}$ and $\partial \check{D}_{2}^{\hat{\sigma} k}$ are depicted in figure 5 and the functions $\alpha_{1}(k)$ and $\left\{c_{j}\right\}_{1}^{5}$ are defined as follows:

$$
\begin{aligned}
& \alpha_{1}(k)=\frac{i}{2 k+\sqrt{-\omega}}+\frac{i}{2 \sigma k-\sqrt{-\omega}}, \quad c_{1}(k)=c_{1}^{(1)}(k)+c_{1}^{(2)}(k)+c_{1}^{(3)}(k), \\
& c_{2}\left(k, k_{3}\right)=-\frac{1}{4 i\left(k^{2}-k_{3}^{2}\right)} \frac{A\left(k_{3}^{2}\right)}{2 k+\sqrt{-\omega}}, \quad c_{3}\left(k, k_{2}\right)=\frac{\alpha_{1}(k)}{4} \frac{A\left(k_{2}^{2}\right)}{\left(k^{2}-k_{2}^{2}\right)}, \\
& c_{4}\left(k, k_{2}\right)=\frac{i A\left(k_{2}^{2}\right)}{(2 k+\sqrt{-\omega})\left(4 k_{2}^{2}+\omega\right)}, \quad c_{5}\left(k, k_{2}, k_{3}\right)=-\frac{1}{16} \frac{A\left(k_{2}^{2}\right) A\left(k_{3}^{2}\right)}{\left(k_{2}^{2}-k_{3}^{2}\right)\left(k^{2}-k_{3}^{2}\right)}
\end{aligned}
$$

with

$$
\begin{aligned}
c_{1}^{(1)}(k)= & \frac{2 k(\sigma+1)}{\left(4 k^{2}+\omega\right)(2 \sigma k+\sqrt{-\omega})}-\frac{1}{2 \sqrt{-\omega}} \frac{2 k-\sqrt{-\omega}}{(2 \sigma k-\sqrt{-\omega})^{2}}+\frac{1}{2 \sqrt{-\omega}(2 k+\sqrt{-\omega})}, \\
c_{1}^{(2)}(k)= & \frac{1}{2 \hat{\sigma} k-\sqrt{-\omega}}\left(\frac{1}{2 k+\sqrt{-\omega}}+\frac{1}{2 \sigma k-\sqrt{-\omega}}\right), \\
c_{1}^{(3)}(k)= & \frac{4 k^{2}}{i \pi\left(4 k^{2}+\omega\right)^{2}}(\log 4-\log (-\omega))+\frac{2 k \log k}{i \pi\left(4 k^{2}+\omega\right)(2 k-\sqrt{-\omega})} \\
& +\frac{2 k \log (-k)}{i \pi\left(4 k^{2}+\omega\right)(2 k+\sqrt{-\omega})}-\frac{1}{i \pi\left(4 k^{2}+\omega\right)}-\frac{1}{2\left(4 k^{2}+\omega\right)} .
\end{aligned}
$$

Proposition 4.2. For $\omega<0$,

$$
\lambda g_{13}(t)=\frac{1}{i \sqrt{-\omega}} e^{i \omega t}+o(1), \quad t \rightarrow \infty
$$

Proof. Using (4.1), (4.3) and (4.4), we can derive (4.9). However, $\lambda \Phi_{22}(t, k)$ contains the term involving $t e^{i \omega t}$. Here, we prove that the contributions from the terms involving $t e^{i \omega t}$ cancel. The coefficient of $t e^{i \omega t}$ in $\lambda g_{13}^{(2)}$ arises from the second term of (4.4) and the relevant contribution is

$$
\frac{4 i \sqrt{-\omega}}{\pi} \int_{\partial \check{D}_{3}}\left(\frac{1}{2 k+\sqrt{-\omega}}+\frac{1}{2 k-\sqrt{-\omega}}\right) d k=-2 \sqrt{-\omega},
$$


where we have used the fact that the integrand has poles at $k=-\sqrt{-\omega} / 2$ and at $k=\infty$. Regarding the coefficient of $t e^{i \omega t}$ in $\lambda g_{13}^{(1)}$, we note that $I_{2}$ for $\omega<0$ is given by

$$
\begin{aligned}
I_{2}(t, k)= & e^{-4 i k^{2} t}\left\{\alpha_{1}(k) \frac{i(2 k-\sqrt{-\omega}) t}{2 k+\sqrt{-\omega}}+c_{1}(k) \frac{e^{i t\left(4 k^{2}+\omega\right)}-1}{i(2 k+\sqrt{-\omega})}\right. \\
& \left.+\frac{2 i \sqrt{-\omega}}{(2 k+\sqrt{-\omega})^{2}}\left(\frac{e^{i t\left(4 k^{2}+\omega\right)}-1}{4 k^{2}+\omega}-i t e^{i t\left(4 k^{2}+\omega\right)}\right)\right\} .
\end{aligned}
$$

Hence, the coefficient of $t e^{i \omega t}$ in $\lambda g_{13}^{(1)}$ arises from the last term in (4.11) and the relevant contribution is

$$
\frac{4 \sqrt{-\omega}}{i \pi} \int_{\partial \check{D}_{3}} k\left(\frac{1}{(2 k+\sqrt{-\omega})^{2}}-\frac{1}{(2 k-\sqrt{-\omega})^{2}}\right) d k=2 \sqrt{-\omega}
$$

where we have used the fact that the integrand has poles at $k=-\sqrt{-\omega} / 2$ and at $k=\infty$. From (4.10) and (4.12), it follows that the terms involving $t e^{i \omega t}$ cancel.

\section{Acknowledgements}

The author thanks A S Fokas for his suggestions and discussions.

\section{References}

[1] A. de Monvel Boutet, A. S. Fokas and D. Shepelsky, Analysis of the global relation for the nonlinear Schrödinger equation on the half-line, Lett. Math. Phys. 65 (2003) 199-212.

[2] A. Boutet de Monvel, A. Its and V. Kotlyarov, Long-time asymptotics for the focusing NLS equation with time-periodic boundary condition, C. R. Math. Acad. Sci., Paris 345 (2007) 615-620.

[3] A. Boutet de Monvel, A. Its and V. Kotlyarov Long-time asymptotics for the focusing NLS equation with time-periodic boundary condition on the half-line, Commun. Math. Phys. 290 (2009) 479-522.

[4] A. Boutet de Monvel, A. Kotlyarov, D. Shepelsky and C. Zheng, Initial boundary value problems for integrable systems: towards the long-time asymptotics, Nonlinearity 23 (2010) 2483.

[5] A. S. Fokas, A unified transform method for solving linear and certain nonlinear PDEs, Proc. Roy. Soc. London A 453 (1997) 1411-1443.

[6] A. S. Fokas, On the integrability of certain linear and nonlinear partial differential equations, J. Math. Phys. 41 (2000) 4188-4237.

[7] A. S. Fokas, A Unified Approach to Boundary Value Problems, (CBMS-NSF regional conference series in applied mathematics) (Philadelphia, PA: SIAM 2008).

[8] A. S. Fokas and A. R. Its, Soliton generation for initial-boundary value problems, Phys. Rev. Lett. 68 (1992) 3117-3120.

[9] A. S. Fokas and A. R. Its, The linearization of the initial-boundary value problem of the nonlinear Schrödinger equation, SIAM J. Math. Anal. 27 (1996) 738-764.

[10] A. S. Fokas and S. Kamvissis, Zero-dispersion limit for integrable equations on the half-line with linearisable data Abstr. Appl. Anal. 5 (2004) 361-370.

[11] A. S. Fokas and J. Lenells, The unified method: I. Non-linearizable problems on the half-line, J. Phys. A: Math. Theor. 45 (2012) 195201.

[12] A. S. Fokas and E. A. Spence, Synthesis, as opposed to separation, of variables, SIAM Review 25 (2012) 291-324.

[13] G. Hwang, The Fokas method: the Dirichlet to Neumann map for the sine-Gordon equation, Stud. Appl. Math. DOI: 10.1111/sapm.12035

[14] G. Hwang and A. S. Fokas, The modified Korteweg-de Vries equation on the half-line with a sine-wave as Dirichlet datum, J. Nonlinear Math. Phys, 20 (2013) 135-157 
A Perturbative Approach for the Asymptotic Evaluation of Neumann Value for the NLS

[15] S. Kamvissis, Semiclassical nonlinear Schrödinger on the half line, J. Math. Phys. 44 (2003) 58495868.

[16] J. Lenells and A. S. Fokas, The unified method on the half-line: II. NLS on the half-line with $t$-periodic boundary conditions, J. Phys. A: Math. Theor. 45 (2012) 195202. 